\title{
CMOS Enabled Microfluidic Systems for Healthcare Based Applications
}

\author{
Sherjeel M. Khan, Abdurrahman Gumus, Joanna M. Nassar, and Muhammad M. Hussain*
}

With the increased global population, it is more important than ever to expand accessibility to affordable personalized healthcare. In this context, a seamless integration of microfluidic technology for bioanalysis and drug delivery and complementary metal oxide semiconductor (CMOS) technology enabled data-management circuitry is critical. Therefore, here, the fundamentals, integration aspects, and applications of CMOS-enabled microfluidic systems for affordable personalized healthcare systems are presented. Critical components, like sensors, actuators, and their fabrication and packaging, are discussed and reviewed in detail. With the emergence of the Internet-ofThings and the upcoming Internet-of-Everything for a people-process-datadevice connected world, now is the time to take CMOS-enabled microfluidics technology to as many people as possible. There is enormous potential for microfluidic technologies in affordable healthcare for everyone, and CMOS technology will play a major role in making that happen.

\section{Introduction}

Microfluidic systems have the ability to scale down the components of analysis systems and manipulate small amounts of fluids, even less than a picoliter. ${ }^{[1]}$ Some beneficial characteristics of microfluidic systems are the efficient consumption of reagents, use of low-cost materials and fabrication methods, high sensitivity and resolution, short analysis times, and multiplexing of processes. ${ }^{[2-4]}$ By integrating microfluidic systems with sensors, actuators, and interface circuitry to develop Lab-On-a-Chip (LOC) technologies, complex sample analysis and informative diagnostics tests can be carried out on an easy-to-use chip acting as a portable laboratory at the point of care (POC) ${ }^{[4-7]}$ Hybrid technologies are used to incorporate

\author{
S. M. Khan, Dr. A. Gumus, Dr. J. M. Nassar, Prof. M. M. Hussain \\ Integrated Nanotechnology Lab and Integrated Disruptive Electronic \\ Applications (IDEA) Lab \\ Computer Electrical Mathematical Science and Engineering Division \\ King Abdullah University of Science and Technology (KAUST) \\ Thuwal 23955-6900, Saudi Arabia \\ E-mail: muhammadmustafa.hussain@kaust.edu.sa \\ Dr. A. Gumus \\ Department of Electrical and Electronics Engineering \\ Izmir Institute of Technology \\ Urla, 35430 Izmir, Turkey
}

The ORCID identification number(s) for the author(s) of this article can be found under https://doi.org/10.1002/adma.201705759.

DOI: 10.1002/adma.201705759 additional parts, such as syringe pumps, control valves, and microscopes, which not only increase the complexity and cost of the system but also create a dependence of the system on a laboratorybased environment. Technology has now advanced so much that there are fully integrated sample-in-results-out chips for genetic analysis, ${ }^{[8]}$ sensors that can detect a handful of molecules, and many other similar systems. ${ }^{[9]}$ Complementary metal oxide semiconductor (CMOS) integrated LOC systems can be developed using CMOS technologies as sensors, signal conditioners, data processing circuitry, actuators, and wireless communication capabilities. ${ }^{[10-17]}$ CMOS is a set of microfabrication and nanofabrication processes, which is used to manufacture semiconductor chips present inside computer, smartphone, or any electronic gadgets. Consequently, these chips are referred to as CMOS enabled or CMOS based chips. CMOS based chips can act as a processor, memory, and digital logic circuitry for data management. In this review, we will discuss how CMOS based devices have enabled both management of data from microfluidic devices as well as sensors and actuators to help complement the functionality of LOC devices. CMOS logics are simple to make, and consume little to no current in idle state. With the advancements in Moore's law we have seen the size of CMOS devices getting smaller and smaller allowing for more functionality to fit in a smaller area. ${ }^{[18]}$

CMOS plays a significant role in the realization of portable and versatile microfluidic platforms by integrating them with microscale sensors, interfaces, and actuators to form autonomous hybrid systems. ${ }^{[10,12,16]}$ Adding interface electronics to the same CMOS chip as sensors, actuators, data processing, and communication capabilities creates a universal platform for LOC technologies. Having signal amplification and noise removal right at the sensor interface without any signal loss makes the device output more accurate and sensitive, and also removes parasitic and mismatch errors. ${ }^{[1]}$ By using CMOScompatible processes, it is possible to interface directly with the readout circuitry to form an integrated circuit system on a single chip. Signal acquisition from a large array of sensors to a single system requires a complex integration scheme, but CMOS microfabrication processes enable the integration of analogue multiplexers with biosensors on small dice. ${ }^{[19]}$ CMOS also has the capability to actuate pumps and valves in microfluidic devices or to directly influence the fluid itself using electric 
and magnetic forces, thereby replacing the bulky pressuredriven positive displacement pumps. ${ }^{[20-23]}$ CMOS technologies have the ability to fabricate such actuators and corresponding control circuitry on a single chip. Smartphones are also powerful tools for complementing and empowering the capabilities of hybrid CMOS-microfluidic systems, especially with their imaging, computation, and communication capabilities. Most smartphone cameras constitute CMOS-based image sensors. Smartphones' imaging and processing abilities can be used to carry out the complex analysis of blood or other bodily fluids and body parts. ${ }^{[24-26]}$

CMOS technology allows the ultralarge scale integration of high-performance electronics in a reliable and cost-effective way. However, their implementation has been on rigid and bulky thin-film-based materials with limited application areas. Recent development in free-form CMOS electronics ${ }^{[27,28]}$ brings new possibilities in microfluidic systems. Instead of using today's uniformly shaped and rigidly structured CMOS electronics, the integration of free-form CMOS with microfluidic devices could completely revolutionize both LOC diagnostics and wearable or implantable healthcare devices. There has been an ever-increasing trend in research being conducted on CMOS based microfluidics since the early nineties because researchers realized that most LOC devices need an on-chip integration with CMOS in order to be able to fulfill their real promise of performing portable devices performing laboratory tasks outside the bounds of a laboratory. Figure 1a shows the trends in publications on microfluidic devices incorporating CMOS based devices. However, there has not been an extensive review to acknowledge the efforts of scientists in this field and emphasize the importance of advancements in CMOS that made possible the vision of LOC device based POC diagnostics and treatment. Back in 1999, Rasmussen and Zaghloul investigated how CMOS based actuators and sensors are being used with LOC devices discussing electrically driven actuators, flow sensors, and micropumps. ${ }^{[2]}$ We have come a long way since then and a plethora of sensors and actuators have emerged. Sawan et al. reviewed CMOS/microfluidics devices in 2010, albeit with a focus confined toward cell-based diagnostic tools. ${ }^{[30]}$ Most recently, Ghallab et al. discussed some applications of CMOS based microfluidics systems, a few packaging techniques and challenges arising from CMOS integration. ${ }^{[31]}$ However, the review largely focused on some of the applications (polymerase chain reaction (PCR), flow cytometers, and dielectrophoresis architectures) without addressing the individual sensors and actuators that constitute these architectures which have been made possible by CMOS integration.

In this article, we underwent a narrative review of microfluidic systems with a particular focus on CMOS technologies. We conducted a comprehensive survey of research articles that incorporate CMOS based devices to act as sensors, actuator, processors, or signal conditioners in a microfluidic device. A qualitative analysis of those articles that formulated a device/ application employing a CMOS based device with an application directed toward healthcare (especially disease diagnostics) is hereby presented. The need and working principle of such a device are discussed followed by a comparison of a different number of techniques being used by several researchers toward a similar application. We categorically discuss the choice of

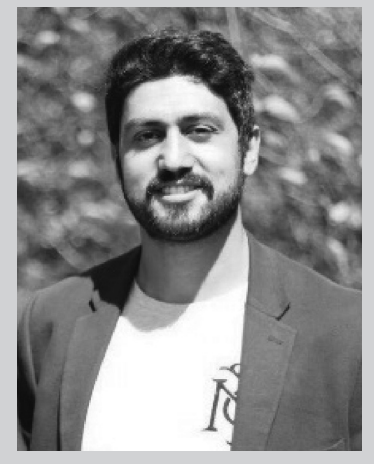

Sherjeel Khan did his Bachelors in Mechatronics Engineering from National University of Sciences and Technology (NUST), Islamabad, Pakistan in 2013. After graduation, he worked as a Design Engineer in a Pakistan-US based startup, Cowlar. He then completed his Master's degree in electrical engineering from Georgia Institute of Technology, USA under Fulbright Scholarship in 2016. He is currently pursuing a Ph.D. in nanotechnology at KAUST under the guidance of Professor Muhammad Hussain.

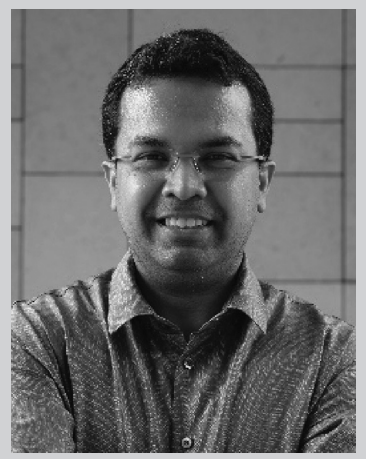

Muhammad Mustafa Hussain (Ph.D., electrical and computer engineering, University of Texas at Austin, 2005) is an associate professor in electrical engineering and $\mathrm{PI}$ of Integrated Nanotechnology Lab. He received his MS from the University of Southern California (USC), Los Angeles and B.Sc. from Bangladesh University of Engineering and Technology (BUET). Previously, he was Program Manager of Emerging Technology Program in SEMATECH, Austin. His research interest is in future-generation electronics and their creative applications.

materials, processes, and devices which are biocompatible, effective, multifunctional, affordable, and manufacturable. We further discuss the integration challenges associated with such devices and present a comprehensive review of packaging techniques used to overcome those challenges. We also present our perspective on the challenges and future trends for next-generation CMOS-microfluidic systems, which include flexible and stretchable integrated CMOS technologies and lowcost paper-based unconventional LOC systems. We outline the role of CMOS technologies and the factors that may affect their deployment in new directions for microfluidic systems. Finally, a number of CMOS based LOC applications and devices are shown that have made a significant contribution toward an improvement in the clinical outcomes.

\section{Microfluidic Systems}

A microfluidic system is composed of channels, valves, chambers, mixers, and sorters working together in harmony to perform a complex set of tasks, predominantly focusing on biological and healthcare applications. ${ }^{[32]}$ Generally, a 

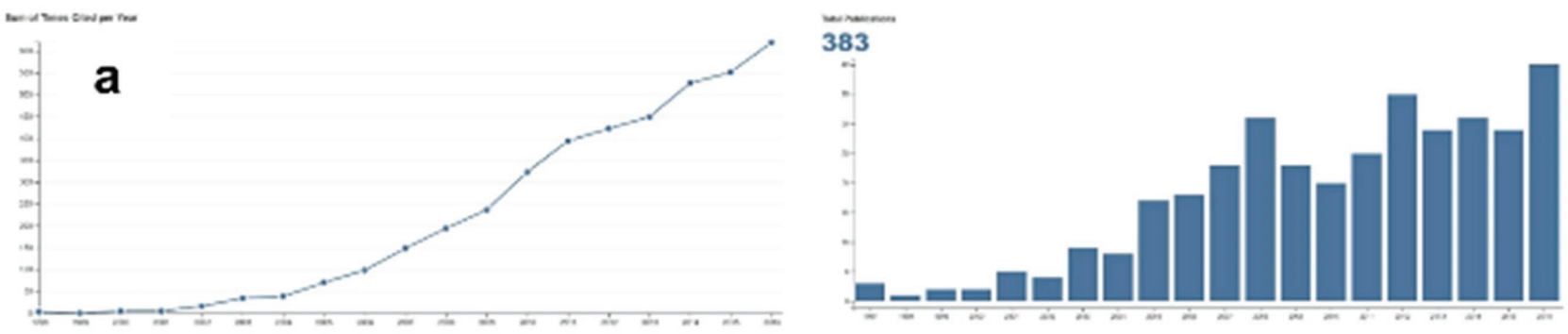

b

(3)

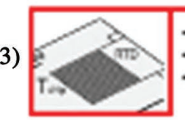

Microheaters (Heater array)
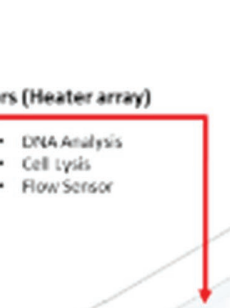
- Cel twis

(1)

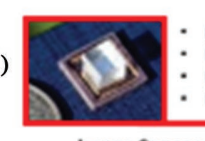
- Particle Datoction
- Fow serso Decuspmeres obtalmiroscose

Image Sensors (CMOS based)

- partide decuction

(2)
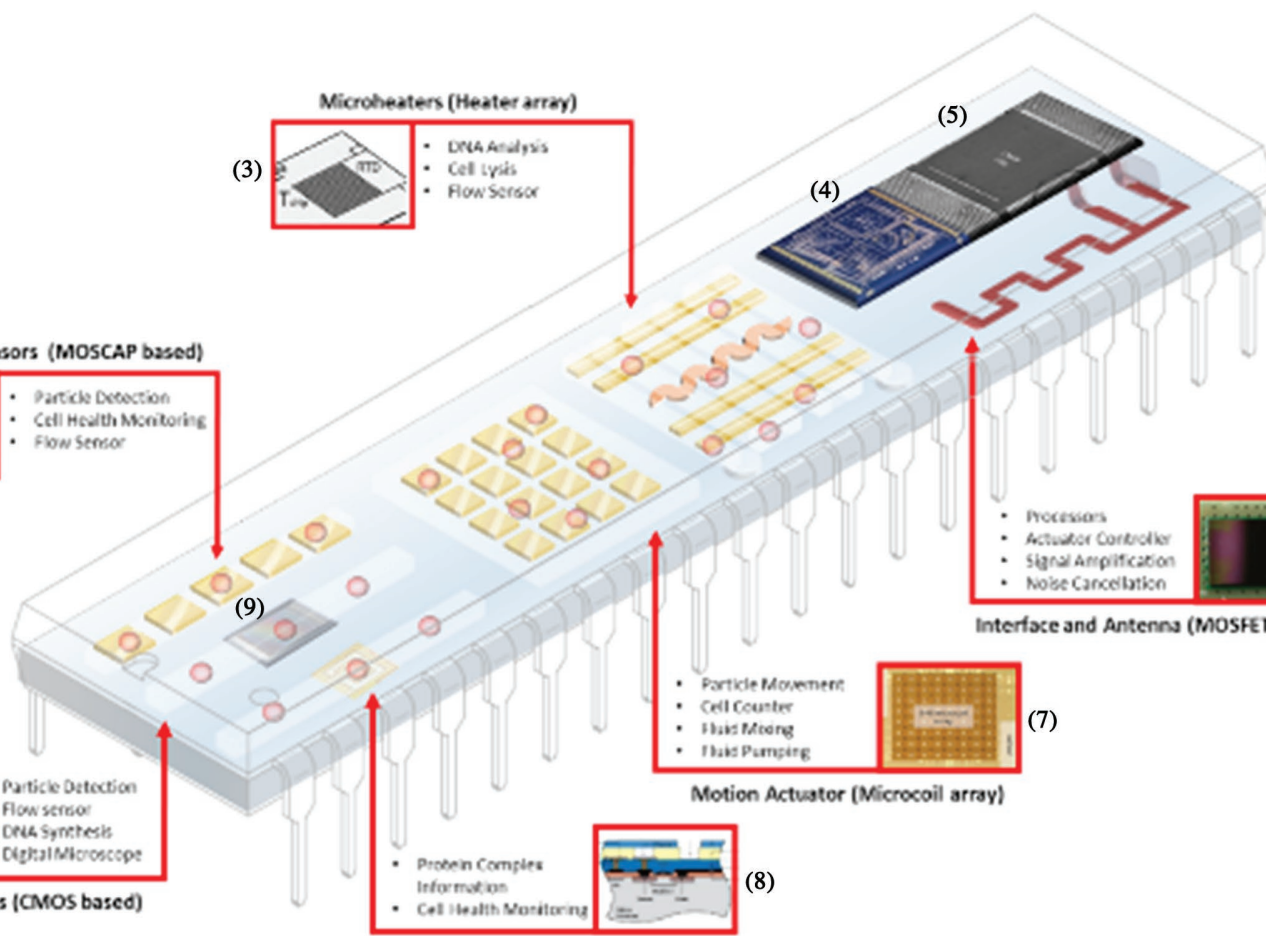
reactions, (ii) reduced analysis costs, where the cost of fabrication is comparatively low while maintaining manufacturability at large scale, and (iii) the ability to make portable POC devices..$^{[2,32,35]}$

\subsection{Fabrication Materials and Processes}

Microfluidic devices rely on the development of simple, reproducible, and mature soft lithography fabrication techniques. Easy-to-use soft lithography-based fabrication procedures are employed to make both poly(dimethylsiloxane) (PDMS)-based microfluidic devices and in line pneumatically activated valves, mixers, and pumps. CMOS-based microfabrication methods, like photolithography, have only been limited to making molds of photoresists for the casting of polymer-based microfluidic devices. ${ }^{[36]}$ However, for applications that demand thermal and chemical stability, microfabrication technologies involving silica and glass are re-emerging as possible candidates. ${ }^{[2]}$

Before the advent of polymers, silicon and glass were the main materials used in microfluidic device fabrication. With time, both faded away as they require complicated, time-consuming, and costly microfabrication methods. Since then, polymers have been the preferred choice owing to their biocompatibility and resistivity to chemicals. ${ }^{[37]}$ Some of the conventional polymeric materials being used today in microfluidics are thermosets (unlike thermoplastics, these materials cannot be remolded by heating) like SU-8, thermoplastic materials like polymethyl methacrylate, and elastomers such as PDMS.[38] Among these polymers, PDMS is the most widely used. Its optical transparency enables optical-based detection techniques, while compatibility with rapid-prototyping has kept the manufacturing costs low. ${ }^{[37]}$ Some of the standard fabrication methods used for polymer-based devices are soft photolithography, ${ }^{[39]}$ stereolithography, ${ }^{[40]} \mathrm{X}$-ray lithography, ${ }^{[40]}$ rapid prototyping (microtransfer), ${ }^{[39]}$ replica molding (micromolding), ${ }^{[39]}$ microinjection molding, ${ }^{[41]}$ hot embossing, ${ }^{[42]}$ photoablation, ${ }^{[43]}$ micromilling, ${ }^{[4]}$ thermoforming of foils, ${ }^{[44]}$ and LIGA (German acronym for Lithographie, Galvanoformung, Abformung) ${ }^{[45]}$ Dry etching ${ }^{[46]}$ and wet etching ${ }^{[47]}$ are mainly used for silica and glass. Using soft lithography as an example, which entails some photolithographic microfabrication methods, gold electrodes are deposited on a polymer following the typical process sequence of mask formation, design patterning through a UV exposure lithography step, photoresist development, gold deposition, and lift-off. ${ }^{[37]}$

Though soft lithography provides accuracy, simplicity, and large-scale production capability, 3D printing has recently attracted attention in the fabrication of modular and low-cost 3D printed microfluidic devices, ${ }^{[48]}$ further empowering the notion of "plug-and-play" microfluidics. ${ }^{[49]}$ The capabilities and accuracies of 3D printers are constantly improving; the first commercial 3D printer specifically designed for creating sealed microfluidic devices is currently on the market. ${ }^{[50]}$ Different methods of 3D printing include extrusion printing, stereolithography (SLA), ${ }^{[51]}$ material, polymer and binder jetting, laser sintering, and laser or e-beam melting. ${ }^{[52]}$ SLA 3D printing uses UV light to cure photoactive polymers (such as resins) in the desired pattern, whereas jetting techniques involve the deposition of photoactive, wax-like, or liquid materials on top of a portable building platform using inkjet printing heads. In sintering, a laser is used to melt and coat layers of plastic powder one on top of another. Melting-based printing techniques follow the same process as sintering, except that a metal powder is used instead of plastic. ${ }^{[52]} 3 \mathrm{D}$ printing is being further explored in resins with properties similar to PDMS, and will soon dominate the fabrication of microfluidic devices due to its high throughput, assembly-free, and low-cost procedures.

\subsection{LOC Systems}

LOC describes a microfluidic device providing a one-chip solution to performing sophisticated analyses that usually require a laboratory. In addition to microchannels, LOC devices need sensors, actuators, and an electronic interface to form a completely automated diagnostic system. Higher yields are achieved by automating and performing laboratory functions on small volumes of liquid in a centimeter-sized chip. Integrated mixers enable controlled separation and mixing which not only helps in disease diagnosis but the possible treatment of diseases.

LOC devices use minuscule amounts of fluid for enhanced sensitivity and lower processing times than the procedures undergone in laboratories. Various approaches to fabricating LOC devices have been tried, as discussed in the previous section, such as integrating the necessary parts of the system on robust and rigid platforms, e.g., glass, silica, and polymers. Elastomers are being investigated intensively for use in microfluidic systems. PDMS specifically is the most commonly used material in the creation of LOC devices, due to its compatibility with the lithography, etching, and deposition processes, hence facilitating the design and integration of micropumps and microvalves for fluid translocation across microfluidic channels. In addition, since PDMS is transparent, this further enables the use of imaging techniques for particle analysis and characterization. However, PDMS is mainly limited to prototyping, as it is prone to aging effects that can cause problems in the consistent functionality and durability of microfluidic devices. To date, one direct competitor to PDMS is thermosetting polymers, which, despite sharing the same appealing physical characteristics as PDMS, are albeit a more expensive alternative.

\section{Role of CMOS in Microfluidics}

Microfluidic devices need to be integrated with electronics to make sense of the data provided by the sensors and they must be able to use that data in an efficient manner. As seen from the previous sections, microfluidic channels need desktop-sized actuators, like syringe pumps, to regulate the fluid flow, and acquired results from such devices need to be interpreted by external cameras and data processing platforms.

Bringing together electronics, micro-electromechanical systems, and microfluidics into one functional system can disrupt the area of personalized medicine and portable sensing. ${ }^{[53]} \mathrm{A}$ smart microfluidic system is composed of (i) sensors to detect 
the desired phenomenon, (ii) actuators to introduce and translate fluids, (iii) microstructures to combine and mix fluids, (iv) filters for purification and separation, and (v) interface electronics to interpret and carry out certain actions based on the acquired data from sensors. These components together form a complex microsystem incorporating mechanical, fluidic, and electronic functions. ${ }^{[7]}$ CMOS-based microfluidic platforms bring together the best of these multiple disciplines to create a unified single-chip platform capable of performing complex biomedical tasks. CMOS has played an integral part in the realization of portable and versatile microfluidic platforms. As it will be seen in the following sections, a tremendous amount of work has been done to integrate microscale sensors, interfaces, and actuators with microfluidic devices using CMOS technology. Several sensors and actuators based on optical, electrochemical, electrical, and magnetic principles have been made using CMOS technology. ${ }^{[10]}$

Integrated circuits (ICs), made by interfacing CMOS technology with microfluidic devices, form what we call a smart system. Sensors may be embedded inside the ICs, while actuators can be controlled by producing electronic or magnetic fields for controlling the movement of objects inside the microfluidic channels. Furthermore, by adding interface electronics on the same CMOS chip, we can build a universal platform for LOC devices. The use of such ICs provides flexibility for making changes or optimizing microfluidic systems without having to alter the physical structure of the device. ${ }^{[1]}$ The beauty of CMOS is that, in a chip much smaller than most microfluidic devices, it can make high-resolution measurements and support large arrays of sensors. ${ }^{[54]}$ CMOS has also shown potential for implementation in applications involving high voltage generation and switching of a high voltage CMOS chip, which allows system miniaturization of the cumbersome high voltage equipment needed for genetic analysis. ${ }^{[55]}$ Figure $1 \mathrm{~b}$ shows an illustration of how different CMOS based technologies can be envisioned to perform multiple functions in a single chip platform capable of performing POC diagnostics.

Impedance measurement techniques are essential for the realization of microfluidic POC diagnostic devices. Developments in CMOS technology have enabled the fabrication of miniaturized impedimetric circuits for the simple, miniaturized, and sensitive detection of genomic signals via a CMOSbased microfluidic device. ${ }^{[56]}$ These advancements in the field of physical and chemical-based sensors have been able to bridge the gap between the electronic and nonelectronic worlds. The next step is to merge microelectronics and micromechanics with microfluidics to build systems capable of not only sensing data but also of performing signal processing, to realize products that can make a real difference. ${ }^{[57]}$ Widespread adaptation of CMOS devices in electronics has been for many reasons, but as with every technology, CMOS comes with some inherent disadvantages; Table 1 discusses some of the advantages and disadvantages of CMOS over the competitive bipolar junction transistor based devices. Despite some of its disadvantages, lower power consumption, large packing density, larger operating voltages, and temperatures have made CMOS the preferred choice over any other technology. The focus of this review paper is how CMOS technology has
Table 1. Comparison of advantages and disadvantages of CMOS over competitive microfabrication technology.

\begin{tabular}{|c|c|}
\hline & CMOS (vs bipolar junction transistor (BJT)) \\
\hline \multirow[t]{6}{*}{ Advantages } & $\begin{array}{l}\text { High input impedance (less leakage current in off state-nA } \\
\text { compared to } \approx u A \text { in } B J T)\end{array}$ \\
\hline & Supports voltages from 3-15 V while BJT work up to $5 \mathrm{~V}$ \\
\hline & Wider operating temperature range $\left(-155\right.$ to $\left.125^{\circ} \mathrm{C}\right)$ \\
\hline & Better noise immunity ( $40 \%$ supply voltage noise margin) \\
\hline & $\begin{array}{l}\text { Smaller devices and better packing density (more devices per } \\
\text { unit area) }\end{array}$ \\
\hline & Ability to integrate with CMOS based sensors with high yield \\
\hline \multirow[t]{3}{*}{ Disadvantages } & Lower switching speeds than BJT ( $1-200$ ns propagation delay) \\
\hline & $\begin{array}{l}\text { Less amplification than BJT (CMOS is not optimized with analog } \\
\text { circuit design) }\end{array}$ \\
\hline & Mismatch between CMOS devices is higher \\
\hline
\end{tabular}

been and can be further merged into microfluidic systems as (i) sensors, (ii) actuators, or as (iii) interface circuitry. Some prominent works and their applications are discussed in the following sections. Figure 2a shows a flow chart summary of the upcoming discussion on the role of CMOS in microfluidic devices.

\subsection{CMOS Enabled Sensors}

Up until the advent of CMOS, optical sensors have been the main source of sensing, of which, microscope and florescent camera-based sensing have been predominant. After a sample is introduced into the channel and passes through several processes in a microfluidic device, it is a common practice that it is then studied under an off-chip optical microscope to make observations and draw conclusions. ${ }^{[2]}$ Another technique is using fluorescence imaging to highlight required parameters in a processed sample and then gather the results using naked eye or image processing. ${ }^{[35]}$ These methods require equipment that is expensive and bulky, thus reducing the application of such systems as POC devices. The only advance in this regard has been the use of smartphone cameras to observe results on the go, which is still a rudimentary technique by modern standards.

CMOS microfabrication techniques brought a revolution to the world of microfluidics. Smart microfluidic systems with integrated sensors are being implemented in most LOC systems which have helped in achieving the desired traits of portability and result accuracy for POC devices. The most significant contribution of CMOS in regard to sensing has come from capacitive and image sensors, which will be discussed in the subsequent subsections, along with various other examples of CMOS-based sensors used in microfluidic devices.

\subsubsection{Capacitive Sensors}

To realize automated microfluidic devices that can carry out liquid sensing and handling, integrated capacitive sensors are needed to determine volume, droplet position, composition, 

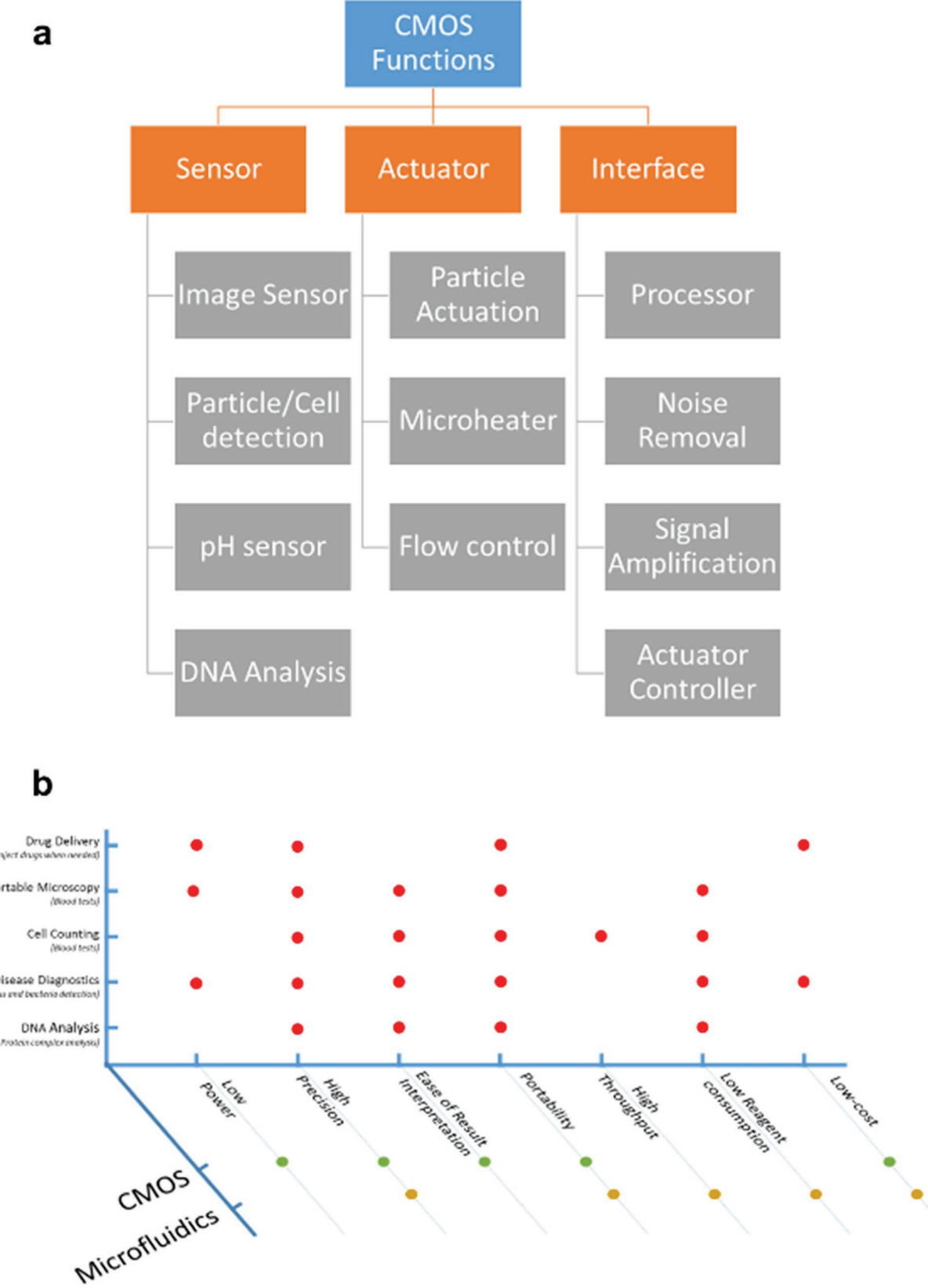

Figure 2. a) Flow chart of various applications enabled by CMOS in microfluidic devices. b) The common and added characteristics of CMOS with microfluidics to form a complete solution for POC diagnostics and other healthcare based devices.

and mass change for volumes in the nanoliter range. ${ }^{[20]}$ For any LOC device, there are two elements of a CMOS capacitive sensor: a sensing layer that can detect capacitance changes in response to variation in certain characteristics of the subject under consideration (e.g., biological quantities), and an on-chip capacitive sensor to measure these capacitance changes. ${ }^{[58]}$

Ghafar-Zadeh et al. and Guiducci et al. have presented several works on DNA synthesis using charge-based capacitive sensors featuring capacitance-to-current transduction techniques. ${ }^{[1,58-61]}$ A voltage pulse is injected into the sensor while measuring the current that is required to charge the unknown capacitance. The current needed for charging corresponded to the value of unknown capacitance. The capacitive sensors were coated with a functionalized layer to immobilize the denatured DNA molecules. The changes in capacitance corresponded to the extent hybridization of the DNA on top of the capacitive sensor. ${ }^{[59]}$ One such CMOS-based microfluidic device for DNA synthesis can be seen in Figure 3a. ${ }^{[1]}$ A syringe is used to inject fluid into the device, and the sensors direct the capacitance changes to a CMOS-based onboard interface for result interpretation. In another study, Romani et al. demonstrated another CMOS-based capacitive $320 \times 320$ sensor array chip for particle detection, fabricated in a $0.35 \mu \mathrm{m}$ standard CMOS process. ${ }^{[62]}$ A similar charge injection technique was used to detect 

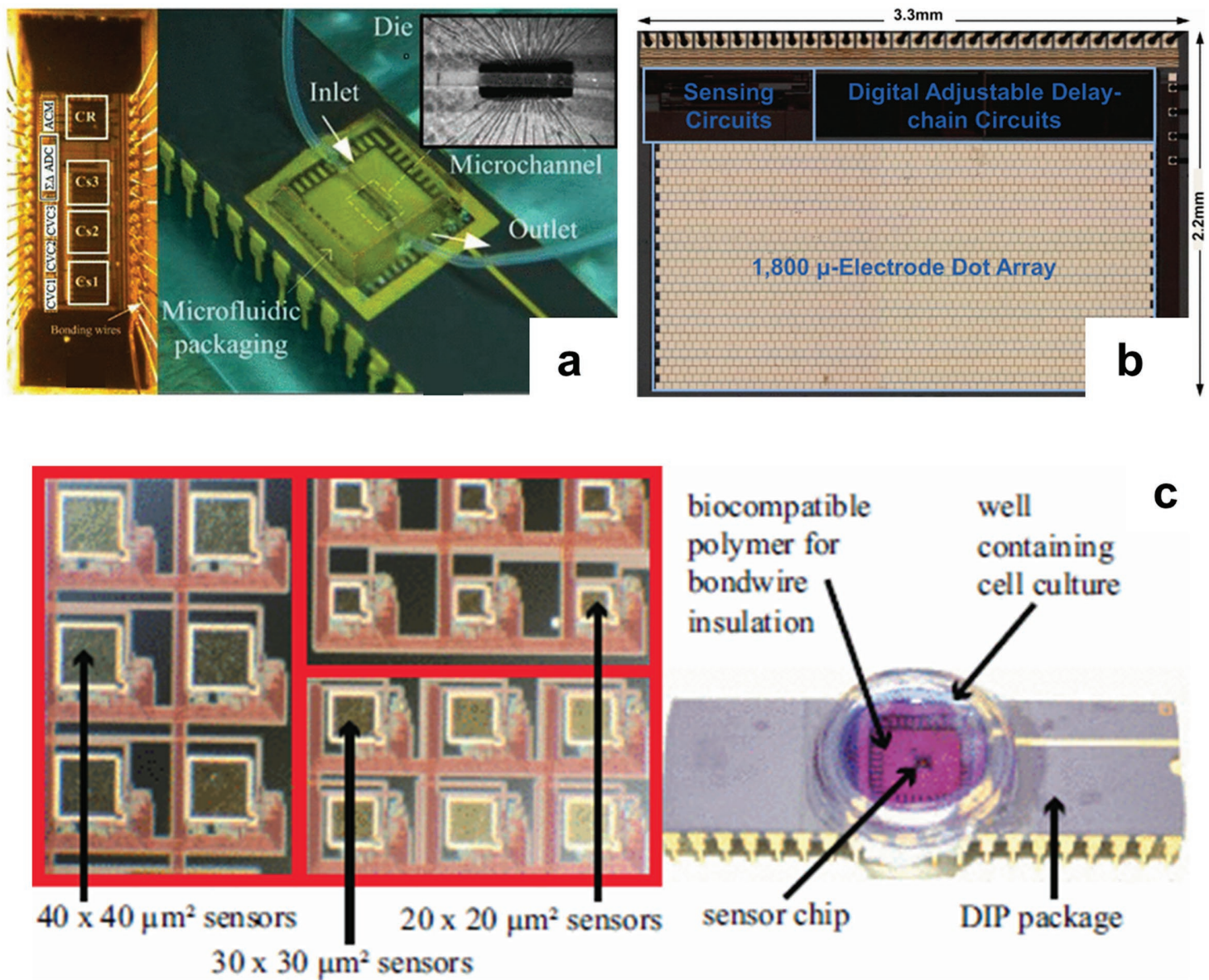

Figure 3. a) Left: Microscopy images of die from the top showing capacitive sensors and interface circuitry. Right: Microfluidic packaging of the chip with a blow-out showing microchannel passing over the sensor chip. Reproduced with permission. [1] Copyright 2008, IEEE. b) Array of microelectrode array with interface circuitry on a single chip. Reproduced with permission. ${ }^{[63]}$ Copyright 2005, IEEE. c) Left: microscopy photo of the fabricated sensors; Right: photograph of the biocompatible packaged version of the chip. Reproduced with permission. ${ }^{[65]}$ Copyright 2016, IEEE.

capacitance variations, which correlate to changes in the dielectric permittivity, after the introduction of bioparticles above the electrodes. The same microelectrode array (MEA) was also used to move particles along the array for multiple particle detections. Such large MEAs are laid out on a substrate, and each pixel is then controlled using row/column decoders. ${ }^{[62,63]}$ The image of one such a chip with MEA integrated with onboard sensing circuits can be seen in Figure 3b. ${ }^{[62,63]}$ Furthermore, Ho et al. demonstrated a microelectrode cell array of microfabricated capacitors that can both actuate a droplet and sense the position of a droplet on the array by employing a real-time feedback control loop to ensure the droplets are moving in the preset fashion. ${ }^{[63]}$ Chen et al. demonstrated another capability of CMOS-fabricated capacitive sensors for sensing a droplet's volume and position. Using feedback from an equally spaced array of electrodes passivated by an $800 \mathrm{~nm}$ layer of $\mathrm{SiO} 2$, they were able to detect the position of a droplet by reading capacitive signals from the linear array of electrodes. Additionally, if the shape of a droplet is known beforehand, the absolute capacitance value can be used to determine the volume of the droplet. The fast time response of $10 \mathrm{~ms}$ can be utilized to instantly measure the rate of mass loss due to evaporating droplets. ${ }^{[20]}$ As discussed before, performing synthesis on droplets enhances speed and sensitivity of tests, thus these devices can be employed to perform tests on a mere blood or urine drop for various disease detection depending upon the particle concentrations.

Monitoring the health of cells is vital to biologists. However, the conventional way of monitoring individual cells under a microscope is a cumbersome process costing time and money. Cell impedance is a good measure of monitoring cell health and growth. CMOS-based sensors have shown potential in observing cell characteristics in a much faster and automated fashion. Welch et al. have demonstrated the use of a capacitive sensor to monitor cell growth with an onboard cell culture. ${ }^{[64]}$ Such devices can enable biologist to monitor cell growth in real time while the cell culture is running. A feat this like was not thought of as monitoring cell growth requires long hours of sitting under a microscope in a lab setup. Prakash et al. used capacitive sensors to detect cell viability. A capacitive biosensor, fabricated with $0.5 \mu \mathrm{m}$ 2-poly 3-metal CMOS technology, was injected with low strength, low-frequency electric fields in a solution containing living cells. ${ }^{[65]}$ The array of biosensors and the packaged version of the chip can be seen in Figure 3c. The 
cells act as insulators and develop dipoles on their membranes. Healthy cells can endure electric dipoles better than weak cells; thus, the obtained value of capacitance is larger for healthy cells. In this case, the capacitance correlates to cell health as it is contingent upon cell morphology, membrane integrity, and extracellular ionic concentration. Such tools can be useful for biocompatibility characterization and medical diagnosis.

Scientists have developed ways of observing certain bioorganisms, like viruses or bacteria, from a diverse population of organisms using functionalized surfaces. Specific organisms get attached to these functionalized biosensor surfaces which open up an opportunity for large-scale automated detection using capacitive sensors. Balasubramanian et al. used a CMOSbased IC platform with embedded capacitive sensors for virus detection using a dummy and a sensing capacitor. The dummy capacitor is used as a reference to find the absolute value of capacitance from the sensing capacitor. An onboard amplifier compared the end values of capacitance to detect the presence of the virus. The virus is first immobilized using an antibody on a silicon wafer. ${ }^{[66]}$ Detection of such a minute quantity of biomaterial (viruses range in size from $20-400 \mathrm{~nm}$ in diameter) was only made possible by using extremely small siliconbased CMOS sensors. Such microfluidic devices have been seen to detect viruses among millions of blood constituent particles.

\subsubsection{Image Sensors}

CMOS-based image sensors are slowly taking over the world of imaging, eliminating the need for charge-coupled devices. The smaller size and lower power consumption while producing higher quality images have been the main drivers of CMOS image sensors' success. Each pixel in a CMOS image sensor contains a photodetector and an amplifier, interfaced with an analog-to-digital converter. CMOS sensors have a faster and more uniform response, and the ability to be fabricated through CMOS processes keeps the price low and allows easier integration with on-chip read-out circuitry.

We are seeing a growing popularity of incorporating CMOS imagers into microfluidic devices. In the years following 2010, tens of research papers are seen on CMOS images sensor integrated microfluidic devices. Gomez-Quinones et al. started with modeling CMOS active sensor that can help in their integration into a microfluidic device. ${ }^{[67]}$ CMOS image sensors are used to plot light spectrums and capture still images. Van Dorst et al. used CMOS-based single-photon counting optical sensors to detect trapped particles in flow cells. The microfluidic channels moved the analyte toward the CMOS optical sensors. They were able to combine an $8 \times 8$ array of photodiodes $\left(500 \times 500 \mu^{2}\right)$, temperature sensors, and a digital interface on a single platform covering an area of $4.3 \mathrm{~mm}^{2}$. Normalized luminescence data from all the samples were created by the interface and saved directly on a universal serial bus (USB), ready to be read on a computer. ${ }^{[68]}$ Jung et al. demonstrated a microfluidic integrated image sensor chip to enable real-time image generation of a sample in the microfluidic chamber of an ePetri (self-imaging Petri dish) platform. ${ }^{[6]}$ They built this system on a commercially available CMOS image sensor by bonding a microfluidic layer to the top of a CMOS image sensor. This device was used for drug tests and cell monitoring applications. Similarly, Singh et al., Misawa et al., and Rodrigues et al. have shown to use chemiluminescence and fluorescencebased CMOS imaging system for that can potentially be used for more efficient and reliable detection of bioparticles. ${ }^{[70-72]}$

Another hybrid CMOS-microfluidic contact image system was demonstrated by Singh et al. They took advantage of the $0.35 \mu \mathrm{m}$ CMOS technology to fabricate small image sensors, and placed them directly underneath microfluidic channels to increase the sensitivity of the system to microlitre volumes of samples. The $128 \times 64$-pixel array was integrated with double sampling amplifiers, and readout circuits on the same chip to form a direct-contact, high spatial resolution luminescencebased optical sensing of chemical analytes. ${ }^{[73]}$ Demircan et al. integrated the CMOS image sensors with microfluidic dielectrophoresis (DEP) channels. A $0.35 \mu \mathrm{m}$ CMOS process was used to fashion a $15 \mu \mathrm{m} \times 15 \mu \mathrm{m}$ active area pixel. Each of the pixels in an array of $32 \times 32$ pixels was then interfaced with reset, buffer, and row switch transistors on the same silicon substrate. The sensor was able to complete cell imaging at high multiplexing rates up to $400 \mathrm{kHz} \cdot{ }^{[74]}$ An illustration of the system and the CMOS enabled device on an Integrated circuit can be seen in Figure 4a,b. Figure 4c shows the resulting cells detected by the CMOS image sensor. All of these works complement LOC devices, which require high-speed real-time monitoring of bioparticles.

DNA synthesis was a milestone, and now we see portable DNA analysis systems thanks to progress in CMOS-based microfluidics technologies. CMOS-based sensors and actuators make it possible to take a single DNA strand, amplify it using microheaters, and analyze it with CMOS-based image sensors. Norian et al. embedded their CMOS-based LOC device with on-chip with single-photon avalanche diodes (SPADs) for fluorescent-image-based monitoring of an ongoing PCR. ${ }^{12]}$ Hall et al. used a single CMOS chip for not only fluorescence imaging based detection but also for high voltage generation. ${ }^{\text {[75] }}$ While DNA synthesis is performed off-chip, we have seen from previously mentioned works ${ }^{[1,58-60]}$ that DNA synthesis can be performed on the same chip to produce a cheap, hand-held one-chip DNA synthesis platform. Eltoukhy et al. presented a system on a chip (SoC), fabricated in a $0.18 \mu \mathrm{m}$ CMOS process, that was fashioned into a LOC capable of sequencing DNA and determining intercellular adenosine triphosphate (ATP) and gene assays. The SoC was loaded with an $8 \times 16$ CMOS image sensor array, 13 bit analog to digital converter (ADC), and column level digital signal processing (DSP). ${ }^{[76]}$

Microscopes have been used by scientists for ages to observe microorganisms. Their operation is time-consuming and they are bulky, which hinders their use in portable devices. Lange et al. and Pang et al. devised a CMOS-based on-chip microfluidic microscope by modifying the design of each pixel to achieve a much higher resolution. ${ }^{[77,78]}$ They created small circular apertures of $1 \mu \mathrm{m}$ on top of each pixel and shone a light on them from the other end. Line traces were created by each pixel as the specimen moved between the CMOS image sensor and the light source. Combining the line traces from each pixel produced an image with a resolution comparable to 

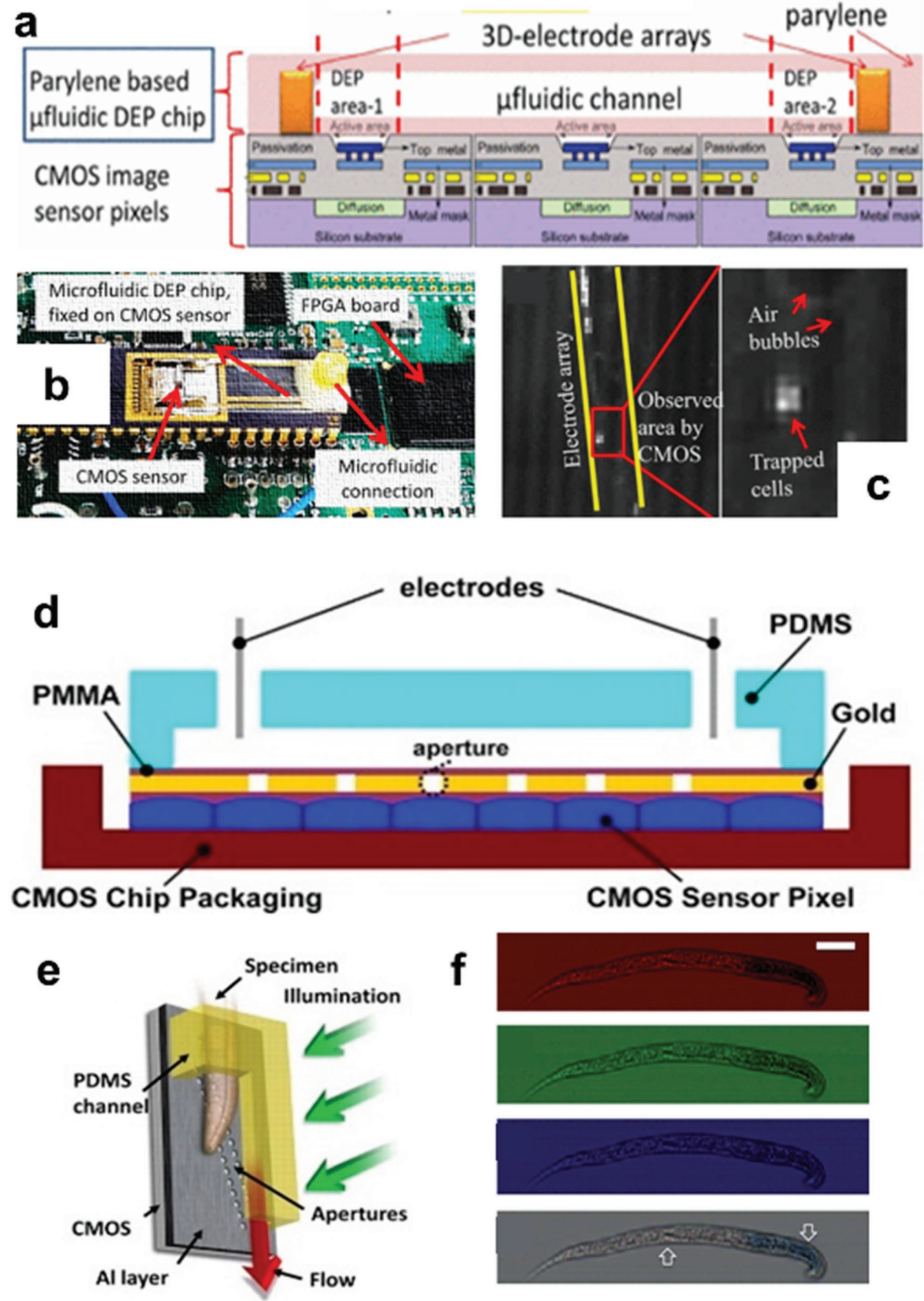

Figure 4. a) Illustration of a microfluidic DEP device on top of a CMOS imager. b) The packaged version of the chip being tested. c) Images of tapped cells in the microfluidic channel obtained by CMOS imagers. a-c) Reproduced with permission. ${ }^{[74]}$ Copyright 2015, IEEE. $d$ ) Cross section view of the device showing integration of CMOS image sensors with microfluidic channel. e) A schematic of gravity driven approach to move specimen over the CMOS sensors; and f) color image reconstruction from individual RGB images. d) Reproduced with permission. ${ }^{[77]}$ Copyright 2010, National Academy of Sciences; e) Reproduced with permission. ${ }^{[78]}$ Copyright 2010, National Academy of Sciences; f) Reproduced with permission. ${ }^{[80]}$ Copyright 2010, National Academy of Sciences.

the aperture size (e.g., $1 \mu \mathrm{m}){ }^{\left[{ }^{[9]}\right.}$ The results were comparable to images obtained otherwise from a traditional microscope. The cross-sectional view and functionality of the device and final results are shown in Figure $4 d-f .{ }^{[77,78,80]}$ The specimen was moved inside the microfluidic channel using gravity while
CMOS image sensors captured red-green-blue (RGB) images to obtain a final colored image. Wang et al. and Yan et al. further demonstrated high $(4 \times)$ resolution and lensless imaging system using CMOS image sensors that are able to detect smaller fast moving bioparticles flowing in a microfluidic channel by 
Table 2. Characteristics of CMOS image sensors used in different applications.

\begin{tabular}{|c|c|c|c|c|c|c|c|c|c|}
\hline Author & Year & Pixel array & Pixel size $[\mathrm{mm}]$ & Resolution [mm] & Process & Size $\left[\mathrm{mm}^{2}\right]$ & Fps [frames s ${ }^{-1}$ ] & Power [mW] & Purpose \\
\hline $\operatorname{Liu}_{[135]}$ & 2017 & $1600 \times 2056$ & 1.1 & $\begin{array}{l}2 \text { (sized particle } \\
\text { detected) }\end{array}$ & $65 \mathrm{~nm}$ BSI CMOS & $5 \times 5$ & 45 & 182.8 & Blood count \\
\hline Van Dorst ${ }^{[68]}$ & 2016 & $8 \times 8$ & 500 & $\begin{array}{l}1-4\left(\mathrm{pg} \mathrm{mL}^{-1}\right. \\
\text { of LLOD) }\end{array}$ & CMOS & 4.3 & - & - & $\begin{array}{l}\text { Sensitive biological } \\
\text { assay readout }\end{array}$ \\
\hline Demircan $^{[74]}$ & 2015 & $32 \times 32$ & 15 & $\begin{array}{c}3 \text { (sized particle } \\
\text { detected) }\end{array}$ & $0.35 \mu \mathrm{m}$ CMOS & - & - & - & Leukemia cell detection \\
\hline Huang ${ }^{[157]}$ & 2014 & $752 \times 548(1.1 \mathrm{MP})$ & 2.2 & $\begin{array}{c}8 \text { (sized particle } \\
\text { detected) }\end{array}$ & FSI-CMOS & $4.51 \times 2.88$ & 60 & $<320$ & Blood count \\
\hline Lee $e^{[158]}$ & 2014 & $3264 \times 2448$ & 1.4 & $\begin{array}{c}8 \text { (sized particle } \\
\text { detected) }\end{array}$ & FSI-CMOS & $4.6 \times 3.5$ & 30 & - & Blood count \\
\hline Jung[69] & 2014 & $2592 \times 1944$ & 2.2 & 0.66 & & $5.7 \times 4.28$ & 14 & 381 & On-chip microscopy \\
\hline Göröcs ${ }^{[159]}$ & 2013 & $2592 \times 1944$ & 2.2 & 5 & CMOS & $\approx 24$ & - & - & On-chip microscopy \\
\hline $\operatorname{Yan}^{[82]}$ & 2012 & $128 \times 96$ & 10 & 2 & $0.18 \mu \mathrm{m}$ CMOS & $2.5 \times 5.0$ & 2475 & - & High speed imaging \\
\hline Wang ${ }^{[81]}$ & 2012 & $128 \times 128$ & 10 & 2.5 & $0.18 \mu \mathrm{m}$ CMOS & $2.5 \times 5.0$ & 1750 & $\begin{array}{c}0.105 \\
\text { (per column) }\end{array}$ & $\begin{array}{l}\text { High resolution } \\
\text { bioparticle imagine }\end{array}$ \\
\hline Singh ${ }^{[70]}$ & 2012 & $64 \times 128$ & 19 & - & $0.35 \mu \mathrm{m}$ CMOS & $2.9 \times 2.7$ & - & 2.3 & $\begin{array}{l}\text { Chemiluminescence } \\
\text { contact imaging }\end{array}$ \\
\hline Misawa ${ }^{[71]}$ & 2011 & $1289 \times 1024$ & 2.5 & $\approx 100$ & CMOS & - & - & - & Portable imaging system \\
\hline Pang ${ }^{[78]}$ & 2010 & $2048 \times 1536$ & 3.2 & 0.8 & CMOS & - & 12 & 240 & $\begin{array}{l}\text { On-chip optofluidic } \\
\text { microscope }\end{array}$ \\
\hline
\end{tabular}

achieving a resolution of as much as $0.25 \mu \mathrm{m}$ at a frame rate of 1750-6000 frames $\mathrm{s}^{-1}$. ${ }^{[81,82]}$

Smartphones can enhance the functionality of POC devices as they provide voltages up to $5 \mathrm{~V}$ that can be used to drive actuators, valves, or any external hardware. ${ }^{[83]}$ Laksanasopin et al. made a cheap smartphone accessory for syphilis detection that was able to perform all the actions generally required in a laboratory-based enzyme-linked immunosorbent assay. ${ }^{[25]}$ Smartphones are loaded with various sensors that can be used in health-related monitoring applications, especially the camera, which makes use of CMOS-based image sensors. ${ }^{[26]}$ Smartphones have the potential of advancing hybrid CMOSmicrofluidic systems where their superior imaging and processing capabilities can be utilized to carry out complex analyses. Araci et al. demonstrated a splendid example of such a system, where a passive microfluidic intraocular pressure (IOP) sensing device was enclosed within the eye socket for glaucoma diagnosis. ${ }^{[84]}$ Changes in the IOP caused the movement of fluids within the microfluidic chambers, which were captured and analyzed using a smartphone camera. Kong et al. achieved a $>13 \times$ magnification using a smartphone and an external lens together when monitoring nucleic acid amplification using fluorescent analysis. ${ }^{[85]}$ Generally, 96-well microplates are used in real-time nucleic acid amplification tests. Obtaining such a high magnification within a small height of $10 \mathrm{~cm}$ enabled them to analyze an $18 \mathrm{~cm}+$ area in one shot, by mapping the fluorescence signals of 25 wells into a $10 \mathrm{~mm}$ wide optical fiber bundle. The fiber bundle was read by the smartphone camera as one single image, thus reading the 25 wells simultaneously without the need for mechanical scanning. Hu et al. have reviewed some portable healthcare platforms using smartphones for cardiovascular disease monitoring. ${ }^{[86]}$ Table 2 shows a summary of different characteristics of several CMOS image sensor based microfluidic devices targeted toward healthcare based applications.

\subsubsection{Biological and Chemical Sensors}

Blood tests are usually carried out by bulky equipment available only in hospitals and laboratories. Kuan et al. recently presented a microfluidic device integrated with CMOS polysilicon nanowire sensors for analyte detection and blood processing. Vortex flow is induced in the microchannels of a micromachined polymethyl-methacrylate based microfluidic device to achieve uniform sample dilution. The CMOS polysilicon nanowires are interfaced with a picoammeter to form a label-free dynamic detection scheme without needing to contain the particles in a chamber. ${ }^{[13]}$ CMOS further enables us to integrate sensors in extremely small-sized areas, which can easily beat the sensitivity and performance of commercially available sensors. Precise knowledge of sample temperature is vital for many biological/chemical tests. Vittoriosi et al. compared the temperature sensing performance of an integrated sensor microfabricated in silicon to a commercially available thermopile. The differential transducers (thermopiles) and reference sensors (RTDs) were both fabricated on silicon. The reference junction of thermopile was at the back side, while the sensing side was on a membrane sitting on top of the microfluidic channel, as shown in Figure 5a. ${ }^{[87]}$ The RTDs were also fabricated to measure the absolute temperature of the chip. After comparing their results to commercial thermopiles, they concluded that IC-based sensors proved more sensitive with a faster time response, albeit being fragile due to a large area of the chip.

Another category of sensors that is important to microfluidics in biological applications is $\mathrm{pH}$ sensors. Hammond et al. 
a

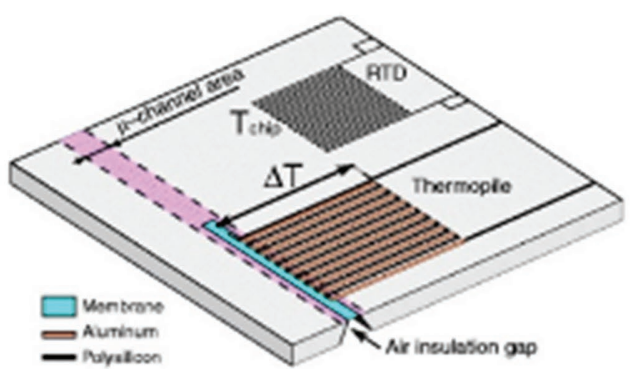

C

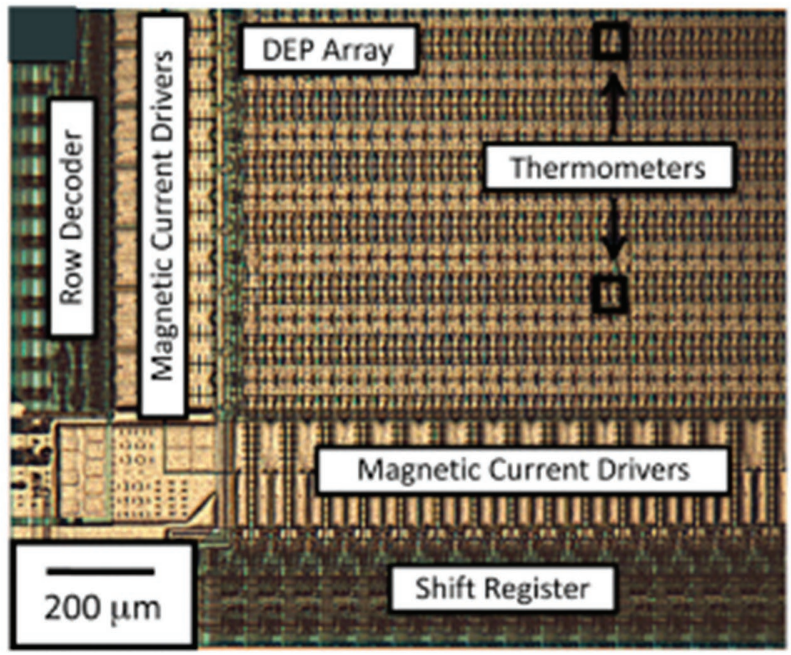

d

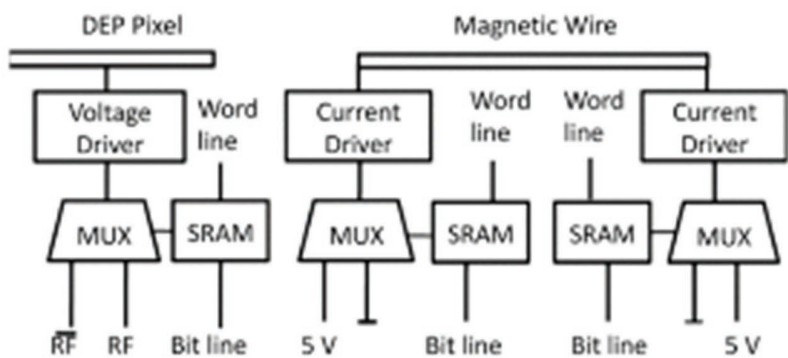

b

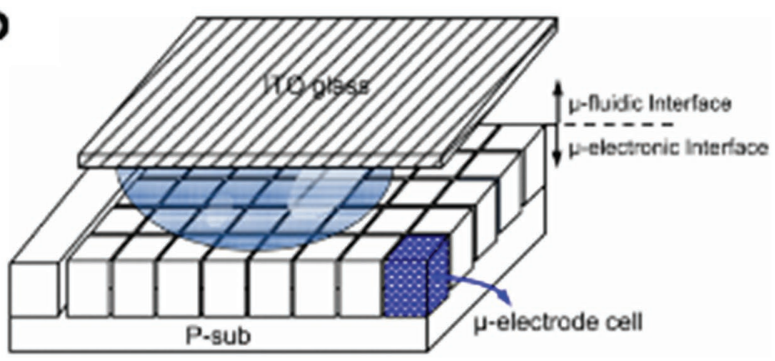

e

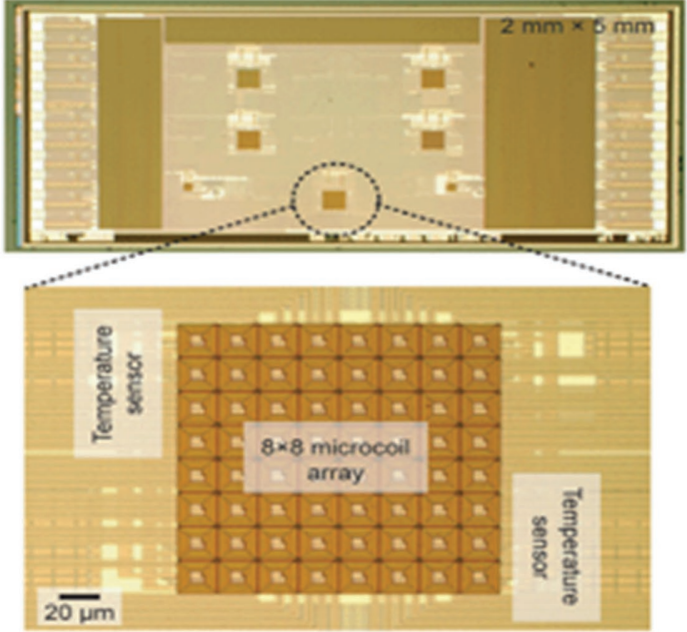

f

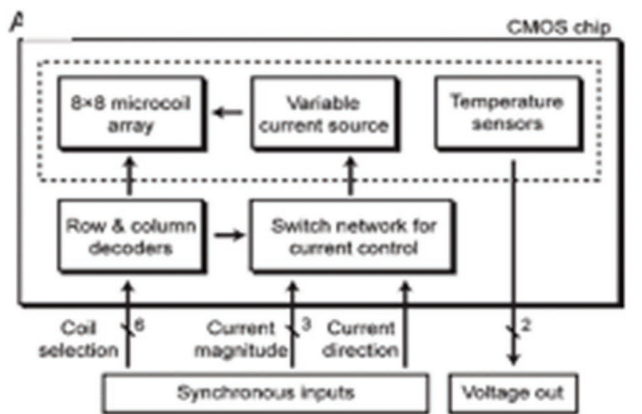

Figure 5. a) An illustration of sensor chip with integrated silicon sensors, membrane, and microchannels. Reproduced with permission. ${ }^{[87]}$ Copyright 2014, Taylor \& Francis. b) A cross-sectional view of the LoCMOS chip showing overlay of microfluidic and microelectronic parts. Reproduced with permission. ${ }^{[63]}$ Copyright 2016, IEEE. c) Microscopy image showing DEP array, magnetic current drivers, thermometer, row decoders, and shift registers integrated on a single chip. d) Schematic of interface circuitry of each DEP pixels and magnetic wire. c,d) Reproduced with permission. ${ }^{[23]}$ Copyright 2009, IEEE). e) Microscopy image of chip with microcoil array. f) Schematic of analog and digital interface circuitry associated with functioning of the microcoil array. e,f) Reproduced with permission. ${ }^{[1]}$ Copyright 2007, RSC.

presented a $\mathrm{pH}$ sensor fabricated using a commercial $0.6 \mu \mathrm{m}$ process. ${ }^{[88]}$ An ion-sensitive field-effect transistor (ISFET) was coated with a $\mathrm{pH}$-sensitive nitride passivation layer. The sensors gave an output voltage according to the $\mathrm{pH}$ of the solution, as the threshold voltage of ISFET was dependent upon the solution's $\mathrm{pH}$. Such sensors fabricated underneath a microchannel can measure the $\mathrm{pH}$ of a flowing liquid. In a different study, a vertically stacked silicon nanowire and ISFET biosensor with suspended microchannels, fabricated in a CMOS-compatible process on silicon on insulator (SOI) substrates, were created by Buitrago et al. ${ }^{[89]}$ The minuscule dimensions of the sensors allowed the handling of nanolitre-scale samples. PDMS with microfabricated channels delivered fluid to the sensors. The sensing areas of the ISFET were exposed to make contact with incoming fluids. Both of these works used ion-sensitive sensing devices, working under the assumption that charged molecules, like DNA or proteins, change the gate potential of FET devices, which can be sensed, as explained by Bergveld et al. and Stern et al. ${ }^{[90]}$ Similarly, Welch and Christen integrated ISFET based $\mathrm{pH}$ sensor, in addition to capacitive sensors, to monitor the $\mathrm{pH}$ of a solution while a cell culture is in process. ${ }^{[64]}$ This provides biologist a deep insight into the condition that affects cell growth in real time. Similarly, Cao et al. have also shown a combination of $\mathrm{pH}$ sensor and an image sensor on a compact single platform using low cost and scalable manufacturing methods. ${ }^{[1]}$ Such devices bring us closer toward making POC diagnostic devices. 
Transistors are generally part of the interface with sensors. However, CMOS-based transistors have also been used as sensors. Protein complexes provide information about the nutritional status of a cell. Kim et al. demonstrated the detection of streptavidin-biotin protein complexes using an extended gate field effect transistor (EGFET)-based biosensor unified with a silicon microfluidic channel. ${ }^{[92]}$ Gold was used as a functionalized layer to bind streptavidin. The reactions between biotin and streptavidin were sensed by computing the flat band voltage shift. The length of biomolecules formed as a result of these reactions. These interactions caused a decrease in the drain current of the EGFET due to the capacitance effect. This reduction in drain current can further be sensed by CMOS based sensors to quantify the growth of biomolecules. This sensor can be easily integrated into a LOC device for real-time detection of various protein molecules. ${ }^{[92]}$ Chang et al. invented a method to fabricate microchannels capable of detecting flow rates without using a high temperature or high-voltage process. Using aluminum electrodes at both ends of a microchannel, they were able to detect a flow rate as low as $9 \mathrm{pL} \mathrm{s}^{-1}{ }^{\left[{ }^{93]}\right.}$ Ability to detect these small flow rates can enable engineers to fabricate devices employing extremely low flow rates to enhance sensing times and reduce the power required to flow fluids. By using CMOS-compatible processes to make such devices, such flow sensors can be easily interfaced with readout electronics to form a single Integrated circuit-system on chip solution.

Chromatography is another important analytical process used in biological processes to separate individual components in a gas/vapor mixture that can be used for environmental monitoring and healthcare diagnostics. Going beyond the expensive and bulky chromatography equipment used in labs, researchers have been able to make portable chromatography systems. Ward et al. monolithically integrated CMOS baseline cancellation circuitry with the nanofabricated chemiresistor array in a microfluidic chamber which was able to perform micro gas chromatography. ${ }^{[94,95]}$ Yeo et al. have reviewed some of the applications of microfluidics in biomonitoring. ${ }^{[96]}$ Integration of such devices with CMOS can bring these devices into the palm of everyone's hand.

\subsection{CMOS Enabled Actuators}

As the CMOS industry keeps improving and maturing, we are able to integrate more and more complex functionalities into a single chip. The small size and lower cost of IC can be made useful by designing hybrid IC-microfluidic chips capable of handling biological and chemical applications. Many works have shown the capability of CMOS to actuate pumps and valves in microfluidic devices or actuate the fluid itself using electric and magnetic forces. These CMOS-based actuators have vast potential to replace the bulky pressure-driven positive displacement pumps, such as syringe pumps. One such actuator is electroosmotic flow. When an electric potential is applied across a channel of ion-containing fluid that has fixed charges on its surface, the fluid starts to flow in the microchannel. ${ }^{[2]}$ Consequently, DEP actuators are used to manipulate bioobjects or fluids by applying an electric field to a microelectrode array. ${ }^{[21]}$ Some recent works on using CMOS as actuators will be discussed in the following text.

\subsubsection{Motion Actuators}

Romani et al. used a $320 \times 320$ capacitive sensor array not only for sensing but also as a DEP-based particle actuator. These DEP forces are generated by providing sinusoidal voltages to the metal electrodes through CMOS transfer gates. ${ }^{[62]}$ Kuan et al. shaped an automatic real-time fluidic control in a blood processing device using programmable microfabricated piezoelectric pumps that mixed blood sample with phosphate-buffered saline. ${ }^{[13]}$ Chen et al. and Gao et al. have shown the dual use of a microheater array of microfabricated capacitive electrodes to also act as thermocapillary droplet-propulsion actuators. ${ }^{[20,22]}$ They were able to use thermal Marangoni force induced by a thermal gradient in a fluidic channel with the functionalized hydrophobic surface to actuate droplets across the array. ${ }^{[22]}$ Most electrophoresis actuators require high voltages applied across the far ends of the actuator, thus making their integration into CMOS a bit of a challenge. Even though some of the CMOSbased high voltage power generators will be discussed in the "Interface" section coming next, Xu et al. showed a low-voltagedriven electrophoresis by driving electrode pairs with lower DC voltages. They made a localized control of electrodes by sensing the location of droplets, and only the electrodes around the droplets were locally controlled. ${ }^{[9]}$ This shows the potential of CMOS technologies to fabricate such actuators and consequently develop their control circuitry on a single chip.

Ho et al. demonstrated a microelectrode cell array (MECA) made in a $0.35 \mu \mathrm{m}$ CMOS process for droplet actuation. The MECA is analogous to a microfluidic channel, thus forming a Lab-on-CMOS (LoCMOS) platform. The CMOS fabrication process kept the size small while making it possible to connect the MECA with extended drain metal-oxide-semiconductor field-effect transistors (MOSFETs), which can actuate droplets more smoothly at the higher $12 \mathrm{~V}$ electrical field. The MECA was able to perform conventional microfluidic device tasks like moving, cutting, and mixing droplets while being integrated with the actuation circuitry to produce a LoCMOS on a single chip, as shown in Figure 5b. ${ }^{[63]}$ A hybrid IC-microfluidic chip made by Issadore et al. employs DEP to manipulate living cells and small liquid volumes, and magnetophoresis to manipulate objects tagged with magnetic nanoparticles. ${ }^{[23]}$ The device has a $30 \times 38 \mu \mathrm{m}^{2}$ array of $60 \times 61$ pixels fabricated in a CMOS high voltage $0.6 \mu \mathrm{m}$ process. Each DEP is interfaced with a temperature sensor, current drive, and static random access memory (SRAM) element. The SRAM elements control the delivery of a radio-frequency (RF) signal to each pixel. ${ }^{[23]}$ An image and schematic of the actuation and interface elements are shown in Figure 5c,d. Lee et al. fabricated a single IC using a $0.18 \mu \mathrm{m}$ Taiwan Semiconductor Manufacturing Corporation (TSMC) process with both analog and digital circuits. The analog part drove the microcoil array used for cell manipulation in the microfluidic channel while the digital part, consisting of field-effect transistor and row/column decoders, performed the task of selecting each coil and then controlling the current in the microcoil. An image and schematic of the actuation and interface elements are shown in Figure 5e,f. ${ }^{[11]}$ Liu et al. further demonstrated the motion actuation technique by attaching biocompatible magnetic beads to biological cells and using a microcoil array in an IC to control the movement of particles. The $5 \times 2 \mathrm{~mm}^{2} \mathrm{CMOS}$ 
chip was fabricated using TSMC $0.18 \mu \mathrm{m}$ technology. The $8 \times$ 8 microcoil array was controlled by row and column devices. ${ }^{[98]}$ This technique provides the flexibility of actuating any particle as long as it can be magnetically tagged.

\subsubsection{Microheaters}

At the heart of DNA analysis, the main process going on is PCR. In PCR, DNA samples are multiplied at different stages, where each step requires a different and precise temperature for efficient DNA analysis. This being one of the most important applications of portable LOC systems requiring precise temperature control while keeping the power consumption to a minimum. CMOS based processes have the ability to provide us with such precise microheaters, which can enable efficient and portable DNA analysis devices. This had been a challenge in the past, but Martinez-Quijada et al. invented a fast, lowpower thermal control which permits millimeter-scaled thermally independent regions. ${ }^{[99]}$ The design was optimized for LOC devices that can be integrated with standard CMOS processes. An aluminum layer stack on a silicon substrate driven by a custom-designed linear voltage-to-current converter acted as the heater. These CMOS-based heaters are essential to portable DNA-testing devices which require accurate temperature control.

\subsection{CMOS Based Interface Circuitry}

Sensors need an electronic interface to perform the function of handheld POC diagnostic devices. These interfaces can not only provide onboard processing for real-time interpretation of results but they also help in noise reduction and amplification which are an integral part of a sensing system. Every sensor needs such signal conditioning circuits before the data can be interpreted by a microprocessor. The integration of sensor and actuators with CMOS based interface circuitry has contributed largely toward the proliferation of LOC devices being used in POC diagnostics. In almost all the works discussed in previous sections, we see a seamless integration of CMOS based actuator and sensors with onboard signal conditioning and processing circuits that allow for a one-chip CMOS die solution that can be employed in a microfluidic platform.

\subsubsection{Data Processors}

Complete SoCs merged with microfluidic systems are now being fabricated using CMOS processes, allowing on-chip digital signal processing, which was previously being carried out by bulky instrumentation modules and desktop PCs. Eltoukhy et al. unified a SoC with a DNA-synthesis microfluidic device. The SoC, along with image sensors, was equipped with digital signal processing and analogue-to-digital converter modules, which allowed them to reduce noise by integrating onto a single chip a low-noise differential, a high-resolution ADC, background subtraction, correlated multiple sampling, and multiple digitization and averaging circuits. ${ }^{[76]}$
Portable biosensors require discretely assembled but often cumbersome analog multiplexers to maintain a stable and reliable signal acquisition from large arrays of sensors. Such interface systems are quite difficult to incorporate into a singular system, but CMOS microfabrication processes have enabled the integration of analog multiplexers with FET-based biosensors in small one-chip dies. Voitsekhivska et al. built an analog multiplexer consisting of 96 analog transfer gates and 32 shift registers with serial interface control pins for data acquisition from a silicon nanoWire FET-based biosensor, as shown in Figure 6a,b. ${ }^{[19]}$ The system was found to deliver sensitive, accurate, and fast measurements, where 32 integrated multiplexers had less than $10 \mathrm{nA}$ leakage currents. ${ }^{[100]}$ Here we see the advantage of large scale integration and low power consumption capabilities of CMOS providing us with a complete portable solution for POC diagnostics. CMOS also helps realize portable systems for the study of membrane protein, which is currently limited by the bulkiness of existing tools. These studies are essential for understanding the structure and function of drug targets. Crescentini et al. devised a microsystem for membrane protein characterization on high performance CMOS electrochemical instrumentation circuits. ${ }^{[54]}$ The electrochemical instrumentation interface enabled the simultaneous electrochemical characterization of multiple array sites in an automated fashion that required minimal user intervention. ${ }^{[54]}$

\subsubsection{Actuator Controllers}

As seen before in Figure 5d,f, actuators need CMOS based integrated digital and analog interface circuitry to drive and control them. ${ }^{[11,23]}$ More complex control circuits are now being investigated as the scale of CMOS keeps improving. Correia et al. demonstrated a full control unit of seven piezoelectric actuators on a single IC chip. The control unit was completely fabricated in a $0.7 \mu \mathrm{m}$ CMOS process. This pumping system could fully replace the conventional bulky syringe pumping systems, and enable a portable one-chip LOC solution for the analysis of biochemical markers in biological fluids. The interface circuitry delivered acoustic waves to the piezoelectric transducer matrix, driven by power amplifiers to achieve fluid pumping, mixing, and heating. ${ }^{[101]}$ The whole system, featuring a control, shift register, scheduler, and an oscillator unit, was composed of 4555 MOSFETs with only 50-220 mA current consumption. ${ }^{[01]}$ CMOS allows the flexibility of developing an interface that can control various kinds of actuators. Microcoils are extensively used for movement actuation. Inherent to their working principle is large power consumption and complex control circuits for controlling the large number of microcoil arrays. Liu et al. demonstrated a low-power control of a microcoil array by connecting all the coils to a common current source with a FET switch. ${ }^{[98]}$ This way, they were able to selectively control each microcoil using just one current source with the help of row and column decoders.

\subsubsection{Noise Removal and Voltage Amplifiers}

Nanopores have been used to analyze DNA and RNA molecules. Passing a strand of DNA or RNA through these nanopores 

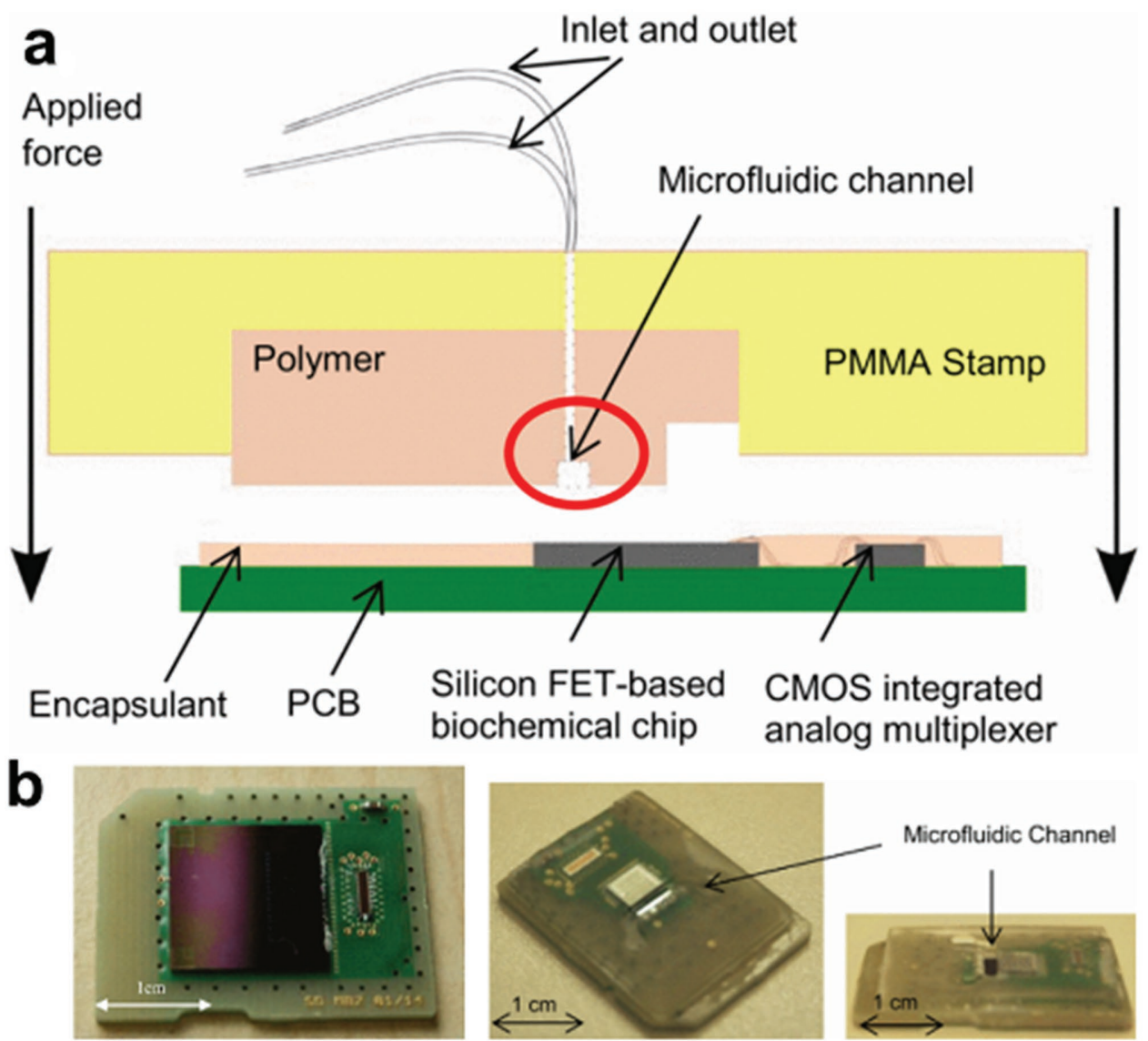

Figure 6. a) Schematic of integration of microfluidic interface with the chip. Reproduced with permission. ${ }^{100]}$ Copyright 2014, IEEE. b) Left: image of chip with interface circuitry and sensors on single chip; Right: image showing microchannel integration with CMOS chip. Reproduced with permission. ${ }^{[19]}$ Copyright 2014, IEEE.

leads to different blocking levels, which can correspond to the characteristics of individual nucleotides. ${ }^{[102]}$ Nanopores are generally biological pores, but being unstable and of fixed size, pose a challenge for CMOS-based microfluidic structures. As we move toward nanometer-scale resolutions in CMOS, solidstate nanopores are now being used for the investigation of several phenomena associated with DNA and RNA proteins. ${ }^{[103]}$ To sense the translocation of individual DNA molecules, Kim et al. fabricated a patch-clamp interface in a $0.35 \mu \mathrm{m}$ CMOS process. Tests were carried out on $\alpha$-hemolysin nanopore with a $1.5 \mathrm{~nm}$ diameter. The microfabricated patch-clamp system performed two functions: it amplified tiny ionic current variations as molecules passed through a nanopore, and nullified the effect of parasitic capacitances. ${ }^{[104]}$ This system can be combined with microfluidic devices for better DNA analysis.

As we have learned from the previous sections, capacitors are the most rudimentary and widely used sensors in CMOS-based microfluidic systems. Control over such sensors has matured and various techniques are being used to convert capacitance to a voltage using CMOS-based electronic interfaces. Romani et al. embedded their sensor array with an onboard charge integrator for capacitance detection and a readout circuit composed of an operational amplifier having common-mode feedback. ${ }^{62]}$ These amplifiers are essential for signal amplification and noise removal as the direct output from sensors is prone to noise, which can make the output indecipherable. Ghafar-Zadeh and Sawan integrated their capacitive sensing arrays with an onchip readout circuit composed of an analog-to-digital converter, an offset cancellation module, and a voltage comparator, using a $0.18 \mu \mathrm{m}$ CMOS process. The novelty of their charge-based capacitance measurement technique removed the effects of parasitic and mismatched errors. The measured current from the sensors was proportional to the capacitance. An on-board switched capacitive amplifier converts this current to voltage, which is compared with a reference voltage using a track-andlatch comparator. This interface circuit can output a bit stream for corresponding capacitance variations, which can be received by any transceiver, thus becoming a complete one-chip solution for DNA synthesis. ${ }^{[1]}$

Electrophoresis-based separation techniques are abundantly used in microfluidic chips but require large voltages, posing a challenge for CMOS integration. However, Khorasani et al. designed a high-voltage microfluidic controller using a DALSA $0.8 \mu \mathrm{m}$ low-voltage/high-voltage (LV/HV) CMOS process, which was able to handle large voltages in electrophoresis-based bioparticle separation systems. ${ }^{[55]}$ The chip included an inductive DC-DC boost converter, four high-voltage output drivers, a voltage divider, and low-voltage control logic. The system was 
successfully able to separate and detect ALFExpress (a DNAsized standard), and now they are developing optical detection using the same CMOS-based DALSA LV/HV microfabrication process to realize a fully integrated, portable platform for genetic analysis. ${ }^{[5]}$ The $3 \times 2.9 \mathrm{~mm}^{2}$ chip, integrated into an IC package, was able to drive the electrophoresis operation in a microfluidic device without assistance. ${ }^{[105]}$ This remarkable achievement showcases the potential of CMOS in overcoming the challenge of executing high voltage operations.

\section{Packaging Compatibility between CMOS and Microfluidics}

Fabrication methods for microfluidic and CMOS-based devices have been refined over several decades. However, when realizing hybrid CMOS-based microfluidic devices, several challenges arise due to differences in scale and processing conditions. ${ }^{[53]}$ The following challenges hinder the growth of CMOS-based microfluidic systems: (i) uncertainties in fabrication and integration processes; (ii) the absence of applicationspecific simulation and modeling software; and (iii) an increase in production costs due to the use of nonstandard production schemes. ${ }^{[57]}$ Another challenge is that integration techniques must lie within the domain of CMOS processes while avoiding the leakage of fluids and their exposure to ICs. ${ }^{[106]}$

Topographical differences exist between the microfluidic channels inside polymer-based microfluidic devices, such as thermoplastics, and the interconnects and CMOS sensors lying on silicon wafers. ${ }^{[10]}$ In order to form reliable interconnects, but also expose CMOS ICs to fluids in microchannels, industrial packaging techniques like the dual in-line package and the flipchip-scale package are used. To tackle the differences in size between the miniature CMOS ICs and the larger microfluidic devices, the ICs are usually affixed to larger substrates, such that the microfluidic device sits on top of the IC. ${ }^{[10]}$ Additionally, to reduce the wasted material from dead volumes during batch fabrication of devices, alignment methods must be very accurate. ${ }^{[7]}$ Finally, the use of such CMOS-based microfluidic systems can only be justified if the cost of production is kept low while offering higher throughput rates. Hereon, we will see how different techniques are used to overcome the barriers of packaging incompatibility between CMOS and microfluidicsbased fabrication processes.

\subsection{Wire Bonding and Flip-Chip Bonding}

Wire bonding and flip-chip bonding are the predominant commercial techniques used to make fast and reliable interconnects between ICs and packages, which is why they have also been used to make interconnects in CMOS-based microfluidic devices. Uddin et al. used wire bonding to connect their hybrid CMOS-microfluidic system to a printed circuit board (PCB), ${ }^{[53]}$ while Wu et al. bonded a CMOS-based microfluidic device to a thin PCB using flip-chip bonding. ${ }^{[107]} \mathrm{How}$ ever, these methods have several shortcomings. Wire bonds cannot be insulated using current microfabrication techniques, which make them prone to corrosion, but the flip-chip method requires several preprocessing steps and extra materials that increase costs. ${ }^{[106]}$ More efficient and innovative integration methods for CMOS-based microfluidic devices are available, some of which are discussed in the following text. These methods make faster and more efficient LOC devices by reducing the distances between the target and sensing modules. ${ }^{[108]}$ Furthermore, the seamless integration of sample manipulation with electronic detection in a small package facilitates one-chip solutions for applications such as impedance spectroscopy, electrochemical detection, and magnetically tagged particle detection or manipulation. ${ }^{[107]}$

Screen printing is better than wire bonding in overcoming topographical differences, as it can deposit thick layers of metal. Recently, Yin et al. took advantage of screen-printed metallization to form thick layers of interconnects, overcoming their topographical differences without using wire bonding. A screen-printed mask facilitated the formation of interconnects between the CMOS die and the carrier. A layer of parylene was deposited on top to insulate the CMOS die from fluids while allowing the biosensing electrodes to remain exposed and in contact with the fluid. ${ }^{[106]}$ Ghafar-Zadeh et al. followed a similar method by printing complex 3D microfluidic structures using the direct-write fabrication process (DWFP). A mixture of petroleum jelly and microcrystalline ink was deposited through a micronozzle. ${ }^{[14]}$ DWFP was carried out in three steps. The deposited ink was encapsulated by an epoxy. After curing the epoxy, the ink was removed, creating a microchannel on top of the previously fabricated capacitive sensing electrodes. ${ }^{[109]}$ These structures were used to create microchannels in the microfluidic device using soft lithography. The sensing electrodes, integrated with the microfluidic device, were connected to a PCB using wire bonding. DWFP, being a low-temperature process, is fully compatible with CMOS processes. Additionally, microfluidic channels can be printed directly on top of an IC. . $^{[10]}$

Halonen et al. combined flip-chip bonding with low temperature co-fired ceramic (LTCC) technology to make a cellculture-compatible package for CMOS-based microfluidic devices. The pads of a sensor chip were wire-bonded with gold, and then the wires were manually removed, leaving only small gold bumps on the pad. On the carrier side, conductive lines were printed on the LTCC packages and a conductive adhesive was applied to the contact pads. The sensor chip pads with gold bumps were then aligned with epoxy-laden pads on the LTCC carrier using a flip-chip bonder, and this package was cured at $150 \mathrm{oC}$ to secure the contacts. The gap between the chip and carrier LTCC was sealed using an epoxy underfill. ${ }^{[11]}$ The schematic of the fabrication sequence is depicted in Figure 7a,b.[111] Welch et al. and Wu et al. employed a similar technique based on flip-chip bonding using a solder bump, an epoxy seal, and a heat reflow process to make electrical connections between the interconnects on a microfluidic device and the metallic pads on an IC. ${ }^{[15,107]}$ In both of these techniques, microfluidic channels on top of the IC delivered fluid to the sensing area of the chip, and the sensors were connected to the IC using the previously discussed integration techniques (i.e., the formation of electrical connections). As the last step, the systems were sealed to avoid fluid leakage using an epoxy. Welch et al. demonstrated this concept on a 


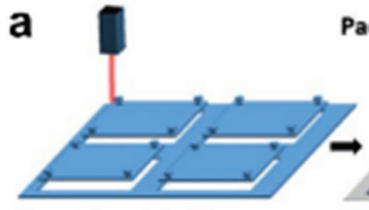

Green sheet blanking: laser pattern

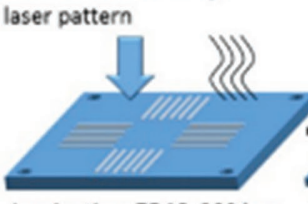

Laminating: $75^{\circ} \mathrm{C}, 300$ bar

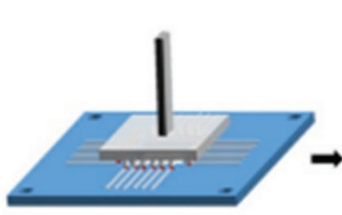

Alumina stamp for silver epoxy "bumps" on LTCC contact pads
Package manufacturing process

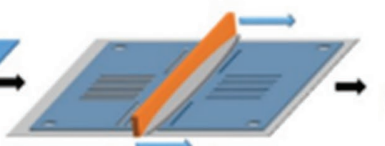

Screen printing conductors (silver paste)

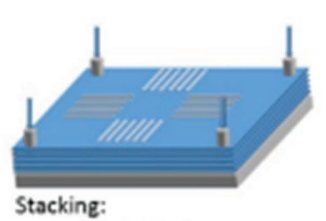
mechanical alienin
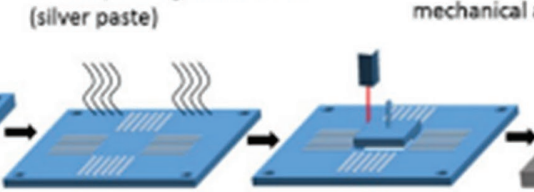

Separate packages and cut holes by laser.

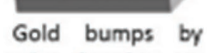
wire bonder on contact pads.

Figure 7. a) Schematic of isotropic conductive adhesive stamping process, sensor chip mounting, and under-fill application. b) Several views of LTCC packaged version with integrated microfluidic channel. a,b) Reproduced under the terms of the Creative Commons Attribution License. ${ }^{111]}$ Copyright 2016, Beilstein Institute. c) Isolated electrical interconnects from the microfluidic channels embedded with IC. Reproduced with permission. ${ }^{[15]}$ Copyright 2013, Institute of Physics.

flexible polyimide PCB as shown in Figure 7c. ${ }^{[15]}$ The microfluidic channel integrated chips interconnects were fanned out on the flexible PCB for easy connections while being isolated from fluids.

\subsection{CMOS Compatible Microchannel}

Dielectrophoresis channels, as shown in previous sections, are made with CMOS-compatible processes, thus allowing uncomplicated integration with CMOS-based sensors. Demircan et al. were able to integrate a dielectrophoresis channel with CMOS image sensors using only four masks. The method claims to be fully compatible with CMOS processes. ${ }^{[74]}$ Deep reactive-ion etching (DRIE) enables the creation of $100 \mu \mathrm{m}$ deep channels on a silicon wafer for applications that require close contact between sensors and fluids. ${ }^{[112]}$ Close contact is essential for impedance spectroscopy at higher frequencies. Kaynak et al. presented the concept of a bipolar junction transistor and the CMOS transistor in a single integrated circuit device platform, wherein a wafer containing circuits and microfluidic channels bonded to another wafer possessing fluid inlet and outlet. The channels formed using DRIE process allowed close contact between $120 \mathrm{GHz}$ dielectric sensor and the fluid because a close contact is essential in impedance spectroscopy at higher frequencies. ${ }^{[13]}$

Most microfluidic devices are fabricated on top of a glass carrier using soft lithography processes. Li et al. proposed a process flow for the transfer of a silicon-sensing die on top of a glass wafer for seamless integration of CMOS sensors with microfluidic devices. ${ }^{[114]}$ The sensing side of a silicon chip is attached to a temporary glass holder using a photoresist. Another glass holder is attached to the back surface of the chip using an epoxy. The temporary glass holder is then released by removing photoresist with acetone to expose the sensor side of the chip. A Ti/Cu film was then deposited on the silicon chip to spread the interconnects to the edge of the carrier wafer. ${ }^{[114]}$ Microfluidic channels were then molded on top of this structure for the electrochemical sensing of fluids. Several other approaches to overcome the size difference between CMOS sensors and microfluidic devices have been tried. Muluneh and Issadore applied three graded layers of PDMS to a millimeter-sized IC for the streamlined delivery of fluids. The fluid delivery from one layer of PDMS to another was enabled by micromachined through-holes. The first layer, which was millimeter-sized and molded with microfluidic channels, was bonded directly to the IC. ${ }^{[115]}$ The second, middle layer of PDMS, which was centimeter-sized with coinciding through-holes, was spun on top of the first layer. Finally, the third layer, which was engraved to allow contact with the environmental fluid, was coated on the top of the second layer. The flow of the process can be seen in Figure $8 d-f^{[115]}$

\subsection{Homogeneous IC integration}

Huang et al. overcame the size difference in CMOS-based photolithography with a die carrier, by leveling the CMOS chip and the carrier surface within a $100 \mathrm{~nm}$ range. This leveling is essential for successful photolithography-based processing, and for good electrical and fluidic continuity. The result was a flat and smooth surface capable of undergoing CMOS-compatible deposition techniques to form interconnects between the CMOS chip and the microfluidic device. ${ }^{[10]}$ Gaps between 


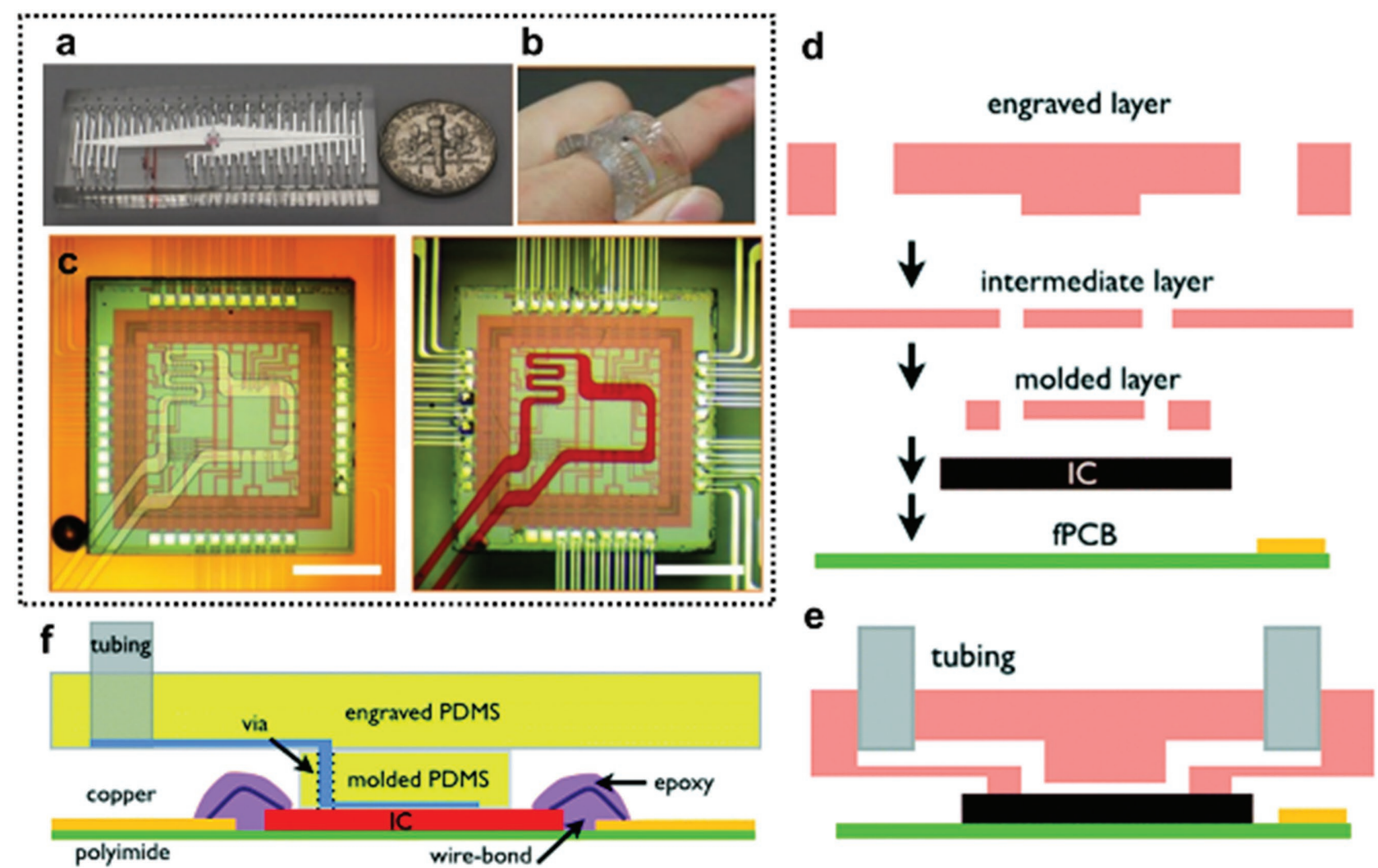

Figure 8. a) PDMS packaged IC with microfluidic channels. b) Bendability of system demonstrated. c) Microscopy image showing channels aligned with CMOS IC before (left) and after (right) liquid metal injection. a-c) Reproduced under the terms of the Creative Commons Attribution License. ${ }^{[115]}$ Copyright 2013, Macmillan Publishers Limited. d) Different layers of PDMS aligned and bonded using stamping-baking technique, e) a cross sectional illustration of the bonded device; and f) an illustration of the fabricated device via to deliver fluid to the IC. $d-f$ ) Reproduced with permission. ${ }^{[115]}$ Copyright 2014 , RSC.

the CMOS chip and the carrier were filled by EPO-TEK 302-3M epoxy, owing to its low shrinkage, low viscosity, lowtemperature curing, and possible removal through plasma dry etching. ${ }^{[10]}$ Alternatively, Uddin et al. achieved a chipspecific wafer integration of an IC on top of a carrier by fabricating sockets in the wafer that were $5 \mu \mathrm{m}$ larger than the IC on all sides depicted in Figure 9a. The IC chips were bonded inside these sockets using an epoxy. They demonstrated this concept by fitting a biosensing chip, fabricated with AMI Semiconductor's $0.5 \mu \mathrm{m}$ CMOS technology, into a socket through adhesive bonding with benzocyclobutene. The lift-off process was then used to pattern interconnects from the micrometer-sized pads on the IC to the centimetersized pads on the carrier wafer, as shown in Figure 9b,c. ${ }^{[53]}$ The potential of merging a complex application-specific integrated circuit (ASIC) with a microfluidic network was shown by Schafer et al. ${ }^{[116]}$ They etched off the top metal layer of AlCuSi from an ASIC, as it was incapable of making an electrical connection in a fluidic network. Pt, $\mathrm{Cr}$, or $\mathrm{Au}$ was then deposited using plasma vapor deposition. Microcapillaries were fabricated on top of the sensing area and coated with a glass cover. Finally, a hydrogenated amorphous silicon (a-Si: $\mathrm{H})$-based color sensor was fabricated on top of the glass using plasma-enhanced chemical vapor deposition. An illustration of the sensor and image of the final device is shown in Figure 9d,e. This colorimetric sensor array was used to analyze samples separated by capillary electrophoretic, presenting a miniaturized application-specific chemical analysis system with integrated electronics. ${ }^{[116]}$

Datta-Chaudhuri et al. have extensively reviewed the challenges faced by SoCs associated with the design, packaging, and integration of systems that interface CMOS with biology and fluidics. These include electrochemical effects, nonstandard packaging, surface treatments, sterilization, microfabrication of surface structures, and microfluidic integration. ${ }^{[16,117]}$ They further discuss a number of approaches used for packaging CMOS dice in LOC systems. ${ }^{[17]}$ Figure 10 shows an illustration of those techniques.

\subsection{Physically Flexible Devices}

Flexible electronics market is expected to be valued more than $\$ 73$ billion by 2027.[118] Polymer-based microfluidic devices are inherently flexible, and thus, if merged smartly with electronics, this multibillion market can be tapped into. Das et al. have opened doors to an emerging field where we are transforming our rigid devices onto flexible platforms for a more conformal and seamless integration particularly with our skin. They used multiple CMOS ICs to create sensing platform with flexible interconnections in a soft polymer assembly. ${ }^{[19]}$ Such packaging techniques will enable researchers to interface complex electronic interface with microfluidic devices to produce skin 

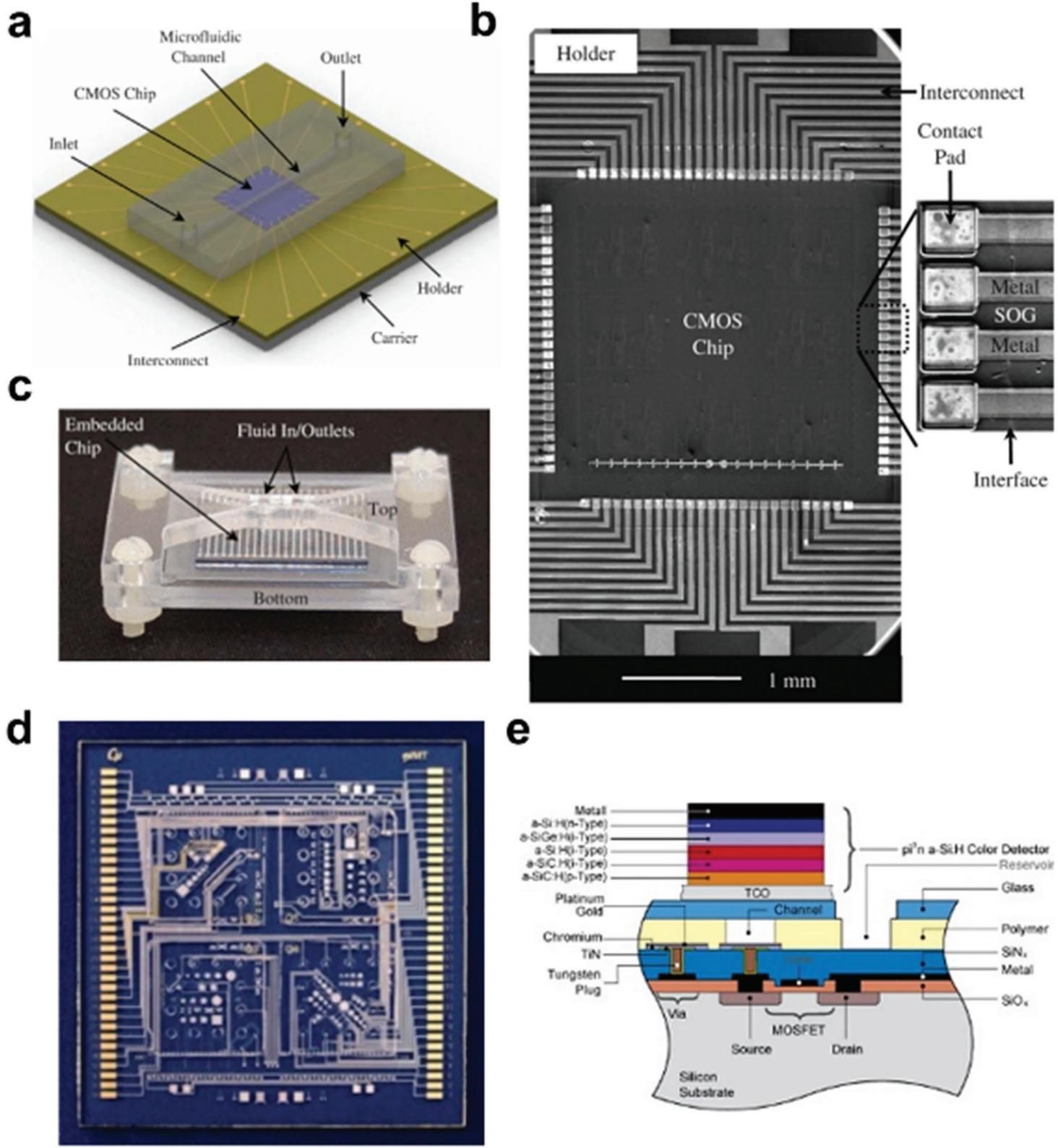

$\mathbf{e}$

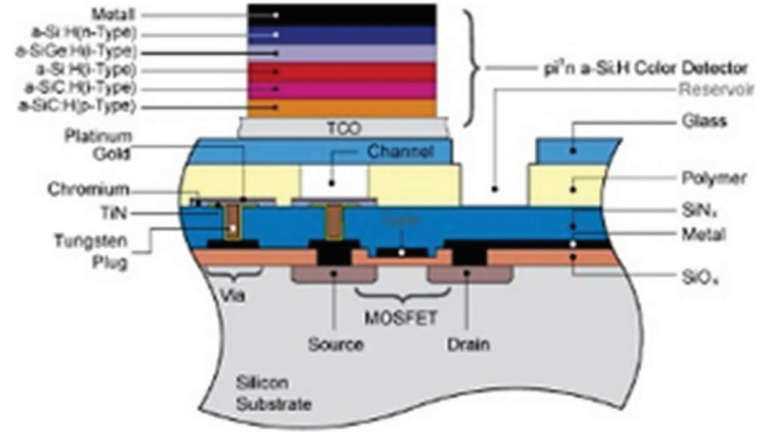

Figure 9. a) Illustration of the system. b) SEM image showing interconnects from the IC. c) A side view of the packaged integrated CMOS microfluidic device. a-c) Reproduced with permission. ${ }^{[53]}$ Copyright 2011, IEEE. d) a-Si:H-based sensors on glass substrate and e) cross section of device showing vias connecting ASIC with fluidic network. d,e) Reproduced with permission. ${ }^{[16]}$ Copyright 2008, Elsevier.

mountable sensing solutions with signal conditioning, processing, and transmission circuits all on a single platform. Welch et al. showed us how an inherently flexible microfluidic device can further be integrated with flexible interconnect without any leakage to form a completely flexible device. ${ }^{[15]}$ Zhang et al. produced a flexible microfluidic device, as seen in Figure $8 \mathrm{a}, \mathrm{b}$, with an IC embedded inside, moving us one step closer toward the realization of point-of-care diagnostics and environmental monitoring devices. ${ }^{[108]}$ The CMOS IC is embedded in one layer of PDMS on which the second layer of PDMS is coated. Microchannels were prefabricated in a second layer using soft lithography. Such devices having CMOS sensor chip with overlaying microfluidic channels, as seen in Figure $8 c,{ }^{[108]}$ provide a huge potential in smart health monitoring devices that are able to transmit continuous real-time data of a person's vital signs to a physician or emergency services.

\section{Applications of CMOS Based Microfluidic Devices}

The fact that microfluidic systems can handle small volumes of liquid makes them ideal for replicating molecular and cellular environments. ${ }^{[32]}$ CMOS-based microfluidic systems have the ability to sample and integrate complex 3D structures of biological tissues. This has paved the way for the realization 
A

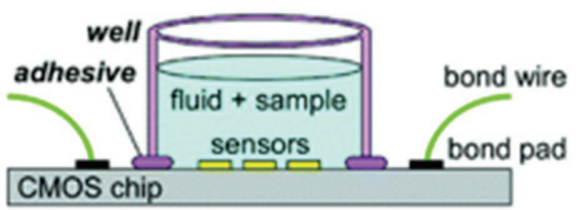

B

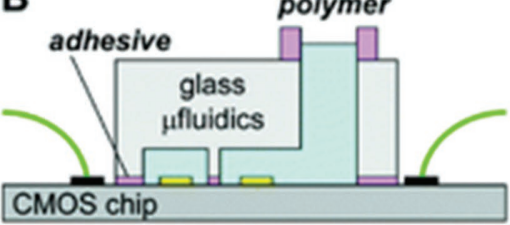

C
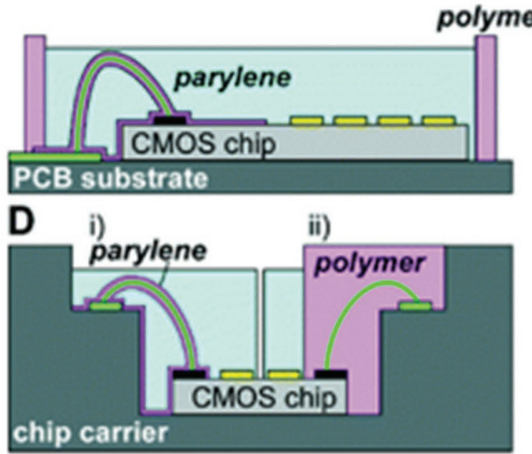

E

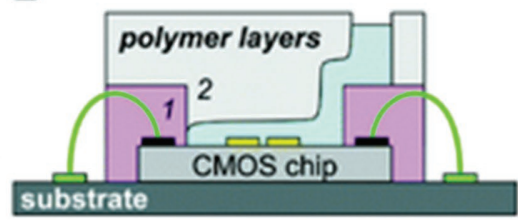

F

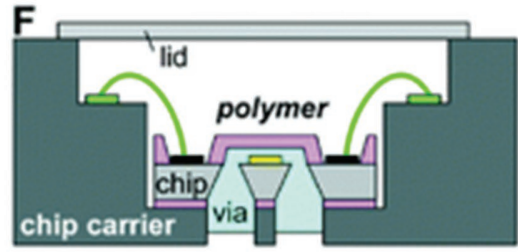

G

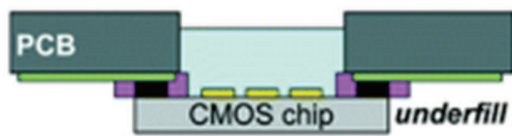

H

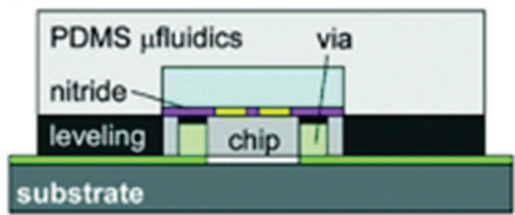

I

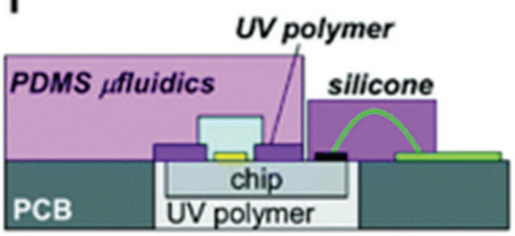

J

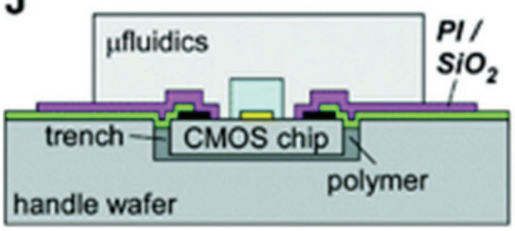

K

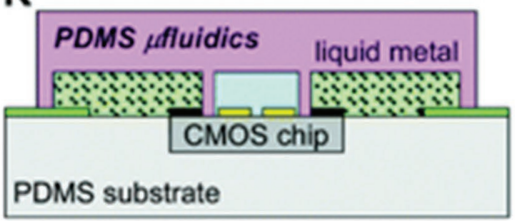

L parylene

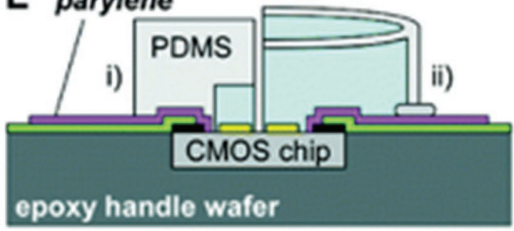

Figure 10. Several packaging techniques for CMOS LOC systems. Pink areas show critical fluid barriers while green areas indicate the protected external electrical connections. Reproduced with permission. ${ }^{[1]}$ Copyright 2014, RSC.

of implantable diagnostic devices and smart wearable systems, which not only diagnose but also possibly treat certain diseases. ${ }^{[120]}$ Integrated LOC devices can combine sophisticated microfluidics with well-developed CMOS-based sensing or analysis. Such devices are label-free, cost-effective, and noninvasive. ${ }^{[111]}$ So far, CMOS-based microfluidic devices are known to work in the following applications: cellular analysis, ${ }^{[121]}$ protein detection, ${ }^{[114,122]}$ nucleic acid detection, ${ }^{[122,123]}$ biophysical analysis, ${ }^{[124]}$ cell separation and sorting, ${ }^{[125,126]}$ material and drug delivery, ${ }^{[127]}$ chip-based drug testing, ${ }^{[128]}$ and biomimetically designed organs-on-chips. ${ }^{[129]}$ Some prominent applications are briefly discussed in coming subsections. Figure $2 \mathrm{~b}$ shows a graph mapping of the different characteristics of both CMOS and microfluidics technologies brought together to form a versatile solution making possible the progress in POC diagnostics. It can be seen that both CMOS and microfluidics bring together the advantages of high precision and portability, and CMOS further enables easier result interpretation and low power portable systems to help form efficient POC devices. Table 3 shows several commercial devices that have been made possible by the amalgam of CMOS with microfluidics to form complete solutions for the healthcare industry.

\subsection{PCR and DNA Analysis}

The ability of microfluidic devices to perform PCR has been a significant breakthrough for POC devices. In PCR, a DNA strand is amplified numerous times for sequencing, cloning, and analysis. ${ }^{[130]}$ LOC devices for real-time PCR can perform pathogen detection for the diagnosis of infectious diseases, genetic fingerprinting for forensics, genetic testing for hereditary diseases, and DNA sequencing for research applications. Norian et al. produced a fully integrated LOC for PCR; integrated CMOS sensors and actuators were able to facilitate temperature control, droplet-based microfluidic transport, and SPAD-based fluorescence measurements. ${ }^{[12]}$ Hatakeyama et al. presented the concept of disposable photodetecting devices for in genetic diagnosis. ${ }^{[131]}$ Thin film transistor (TFT) photosensors were used to detect fluorescent signals from fluorescent-labeled oligonucleotides (short DNA or RNA molecules) immobilized on a titanium oxide layer. Fluorescent-labeled oligonucleotides were hybridized in a microfluidic device to improve DNA-DNA hybridization efficiency. The ability of these devices to incorporate sample preparation, PCR, and subsequent detection into a single automated device reduces handling times and avoids sample contamination. ${ }^{[132]}$

\subsection{Flow Cytometers}

Magnet-activated cell sorting and fluorescence-activated cell sorting are two common techniques used for counting cells in flow cytometers. ${ }^{[126]}$ However, now that CMOS cameras are able to capture up to a billion pixels in one second, they are increasingly useful in flow cytometry devices. ${ }^{[133]}$ Schonbrun 
Table 3. Some commercialized microfluidics platforms employing CMOS based technologies.

\begin{tabular}{|c|c|c|c|c|c|}
\hline Device & Company & Application & CMOS based element & Power source & Reference \\
\hline $\operatorname{mini} P C R$ & Amplyus & Digital PCR & Signal conditioning and amplification & USB & [160] \\
\hline $\min I O N$ & Oxford Naopore & DNA/RNA sequencing & Microchip electrophoresis and signal conditioning & USB & [161] \\
\hline DNA Analyser & Devyser $A B$ & DNA sequencing & Imaging & 3.7 V Li-ion battery & [162] \\
\hline Handheld qPCR machine & Biomeme & PCR & Imaging & USB & {$[163]$} \\
\hline Alere i & Alere & PCR & Imaging & $12 \mathrm{~V} \mathrm{DC}$ & [164] \\
\hline Cobas Liat & Roche Diagnostics & Influenza detector & Imaging and signal conditioning & $15 \mathrm{VDC}$ & {$[165]$} \\
\hline CoaguChek mPOC & Roche Diagnostics & Blood coagulation & Data acquisition, manipulation, and transmission & 3.7 V Li-ion battery & {$[166]$} \\
\hline Accu-Chek Inform II & Roche Diagnostics & Glucose test & Data acquisition, manipulation, and transmission & 3.7 V Li-ion battery & [167] \\
\hline Urisys 1100 & Cobas & Urine analyzer & Data acquisition, manipulation, and transmission & 3.7 V Li-ion battery & {$[168]$} \\
\hline Accutrend Plus system & Cobas & Blood test for cholesterol & Data acquisition, manipulation, and transmission & 3.7 V Li-ion battery & [169] \\
\hline TC20 & Bio-Rad & Automated cell counter & Flow cytometry & $12 \mathrm{VDC}$ & [170] \\
\hline NanoCellect WOLF & Nanocollect & Cell sorter & Flow cytometry & $15 \vee D C$ & {$[171]$} \\
\hline Polyvalent Analyzer (PAx) & ChipCare & Blood test & Data acquisition, manipulation, and transmission & $9 \mathrm{~V}$ battery & {$[172]$} \\
\hline FluorEx & CFT Ltd & Multianalyte immunodetection & Imaging & 3.7 V Li-ion battery & [173] \\
\hline Pathogen detector & DxNow Inc. & Multianalyte immunodetection & Imaging & 3.7 V Li-ion battery & {$[174]$} \\
\hline MPS & Elveflow & Liquid flow rate monitor & Pressure sensor & - & {$[175]$} \\
\hline Electrochemical sensor & microLIQUID & Pathogen and bioparticle detection & Electrochemical sensor & - & [176] \\
\hline
\end{tabular}

et al. presented a multifield of view imaging flow cytometer that can take thousands of images of distinct particles in one second using only a light-emitting diode (LED) for illumination. ${ }^{[133]}$ Dupont et al. fabricated a silicon chip containing a matrix of SPADs to count thousands of fluorescent-labeled cells each minute as they passed through a microfluidic device. ${ }^{[126]}$ Zheng et al. used magnetic sensing to count magnetically tagged cells passing over a microcoil structure in an LOC platform. An impedance sensing and frequency sensing circuit, fabricated in a CMOS $0.18 \mu \mathrm{m}$ process, was able to detect the presence of moving magnetic beads. ${ }^{[134]}$ Most recently, Liu et al. showed a high resolution 3.2 megapixel CMOS image sensor that can be used to count blood cell as efficiently as the bulky lab equipment. ${ }^{[135]}$ This will help us achieve the ability of performing blood test in remote parts of the world which do not have access to laboratories.

\subsection{Disease Detectors}

The POC genetic detection of viruses has applications in food safety testing, environmental monitoring, and clinical diagnostics. ${ }^{[136]}$ In developing countries where illiteracy is more common, there is a dire need for fully automated onsite disease-diagnostic devices that can perform complex laboratory assays in a simplified way at low costs. ${ }^{[137]}$ One such microfluidic POC test device with integrated CMOS-based photosensors, demonstrated by Nayak et al., was capable of detecting Lyme disease. ${ }^{[138]}$ Lyme antigens (toxins that induce an immune response in the body) from a patient's blood sample were immobilized on a microfluidic cassette, then exposed to antibodies and silver amplification reagents, which initiated a sequential binding. This binding caused a decrease in the light from an LED that reached a photodiode at the top of the device, thus indicating the presence of Lyme antigens. An illustration of the detection system can be seen in Figure 11a. ${ }^{[138]}$

Andrianova et al. used a microfluidic device to deliver an analyte to a CMOS-compatible n-channel ISFET, which was able to detect the presence of organophosphorus pesticides. The pesticides were immobilized on the surface of ISFETs and a phosphodiesterase enzyme was used to enhance sensitivity. ${ }^{[139]}$ Demircan et al. designed a label-free LOC detection system that integrated DEP and CMOS imaging for leukemia cell detection. ${ }^{[74]}$ A DEP channel trapped a representative group of cells while CMOS image sensors detected the presence of leukemia cells in the group. El Fouladi et al. flowed magnetotactic bacteria that could attach to a specific pathogenic bacterium along microchannels. Magnetic fields were then used to transfer the bacteria to a CMOS-compatible microfabricated electrode array. Impedance measurements from the respective electrodes indicated the presence or absence of the pathogen. ${ }^{[140]}$

\subsection{Drug Delivery}

Emerging healthcare systems based on microfluidics, aiming to diagnose and possibly intervene in human health conditions, would extremely benefit from integration with CMOS technologies. We believe that flexible CMOS-based microfluidic systems will enable the development of high performance, wearable, and personalized healthcare systems. We envision that microfluidic small-scale pharmaceutical laboratories integrated with CMOS sensors, actuators, and circuits would have the capability to prepare personalized 

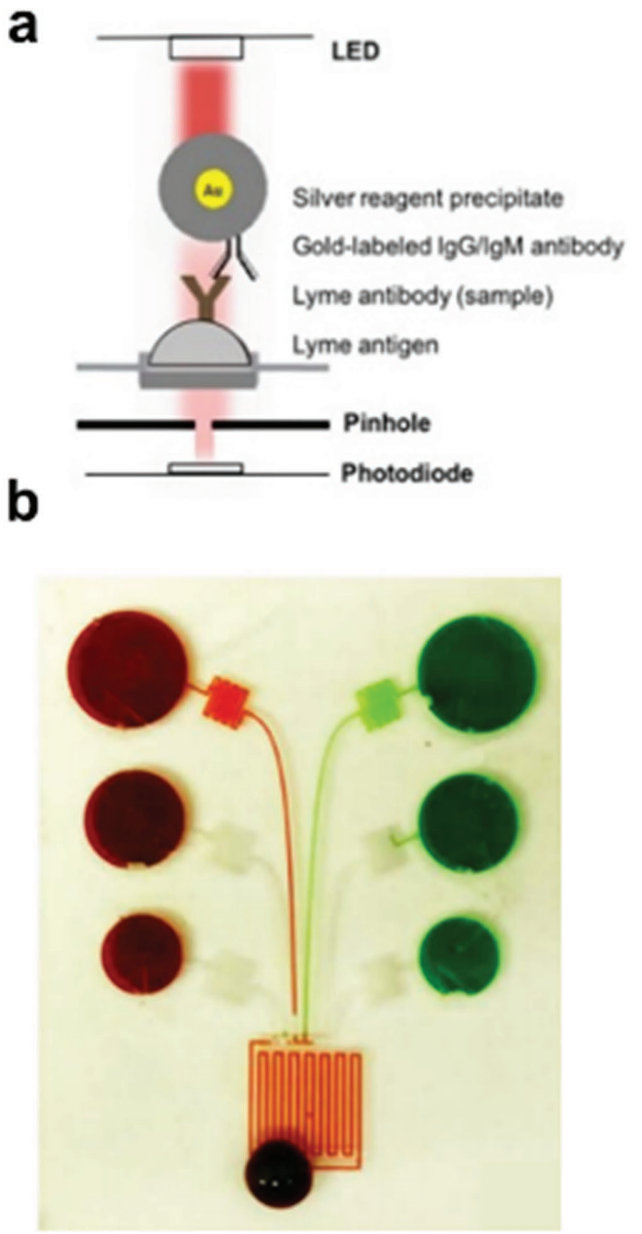

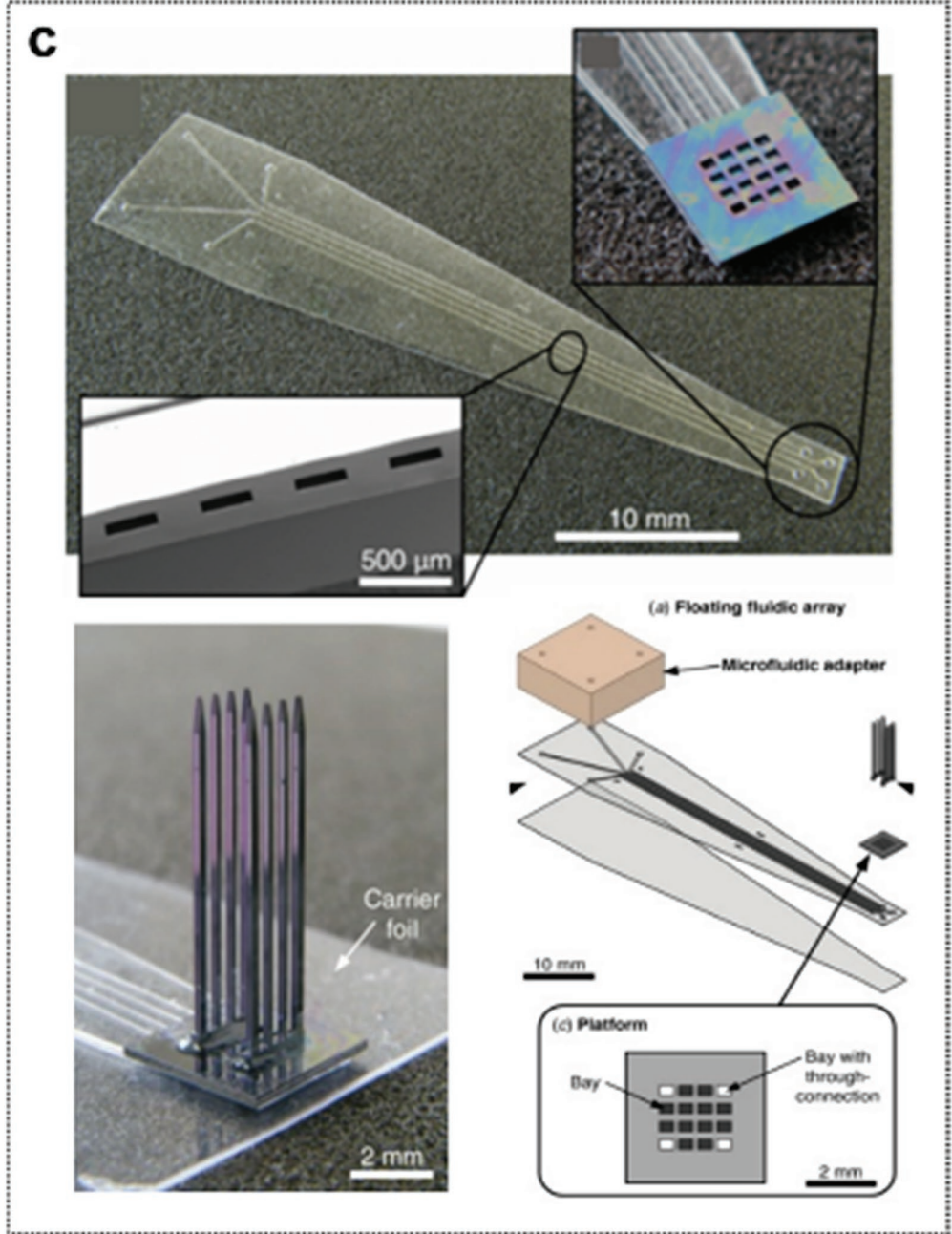

Figure 11. a) Schematic showing how antibody attaches to Lyme antigen and detection using photodiodes. a) Reproduced under terms of Creative Commons Attribution 4.0 International License. ${ }^{[138]}$ Copyright 2016, The Authors, published by Springer Nature. b) Mirror image of the microfluidic small-scale pharmaceutical laboratory integrated with CMOS circuitry. c) 3D floating silicon array attached to a fluidic drug delivery channel. c) Reproduced with permission. ${ }^{[127]}$ Copyright 2011, Institute of Physics.

medicine by mixing different types and dosages of drugs, heating them up to a certain temperature if necessary, and then delivering them to the patient. A system like that could open up new horizons for the personalization of healthcare. The envisioned system, which is currently under development in our lab, can be seen in Figure 11b, where flexible CMOS circuitry is integrated into the back side of the microfluidic channels. This CMOS circuitry can be interface with state-of-the-art drug delivery systems like the one showed by Spieth et al., to materialize the vision of POC devices. The 3D floating silicon array is connected to a microfluidic cable to supply fluids where the drugs are injected into the fluidic adapter through a dosing need as shown in Figure 11c. ${ }^{[127]}$ This delivery system, when integrated with CMOS sensors and actuators, can be controlled to release drugs at regular intervals. Going further, sensors can indicate that a patient is in need of a certain drug which can trigger the actuator to undergo a controlled delivery of the drug through the 3D floating silicon array. Such a system can help us realize the dream of personalized drug delivery. These emerging technologies are likely to have a profound impact on healthcare management and intervention.

\section{Paper-Based Systems}

As we have seen so far, it started with glass and silica being fabrication materials for conventional LOC devices. However, they became less popular than polymers because of their higher fabrication costs and a requirement for greater expertise in microfabrication methods. Even when polymers are used, the functionality of LOC devices demands plastic polymers that have limited to no flexibility. Often the final LOC system is produced in a quite rigid and bulky format, mainly to ensure reliability and intactness of the microfluidic channels over time. Since promising polymers, like PDMS, still have not managed to replace the well-established rigid materials, researchers have been exploring alternative and compatible substrates, such as 
paper. Their goal is mainly to reduce the cost and manufacturing complexity of LOC devices, but unique beneficial characteristics have also been revealed, such as the mechanical flexibility of the LOC platform. Due to its accessibility, flexibility, and desired porosity, which leads to enhanced sensitivity and faster fluid flow (namely, reduced surface tension), paper has been extensively studied and used as a host platform and active material for several types of LoC devices, ${ }^{[141]}$ including sensors, ${ }^{[142,143]}$ actuators, ${ }^{[144]}$ microfluidic devices, ${ }^{[145,146]}$ and health monitoring and diagnostic systems. ${ }^{[147,148]}$ Therefore, interest in paper-based LOC devices has been significantly increasing as they display the potential to be faster, cheaper, and more multiplexed than the current analysis systems. ${ }^{[149]}$

Paper-based 3D microfluidic devices have also shown potential in for overcoming challenges posed by glass and polymerbased microsystems. ${ }^{[150]}$ Microfluidic paper-based analytical devices ( $\mu$ PADs) can be easily integrated into commercial electrochemical readers. Nie et al. demonstrated the compatibility of electrochemical $\mu$ PADs with commercially available glucometers. ${ }^{[151]}$ In another study, they envisioned paper strips being used as cheap and portable diagnostic assays for disease detection and monitoring in remote locations. ${ }^{[148]}$ Paper bestows the $\mu$ PADs with the ability to wick fluids and distribute microlitre-scale samples without external displacement pumps. In that regard, the ultimate platform for a paper-based LOC system is envisioned as a 3D stacked integration strategy, in which paper-based sensors can be vertically interconnected via conductive routings to the rest of the paper-based interface electronics and system components, as tentatively illustrated by Nassar et al. They demonstrated the use of nonfunctionalized cellulose paper in building a large-scale multifunctional "paper skin" platform that integrated six different sensory functionalities: temperature, flow, humidity, touch or pressure, $\mathrm{pH}$, and proximity. ${ }^{[142]}$ Similarly, Whitesides and co-workers demonstrated the development of micro total analysis systems on paper, where printed electronics and microfluidic structures are integrated into a stacking assembly, along with the incorporation of off-the-shelf IC components for system functionality. ${ }^{[145]}$ In a subsequent study, Nassar et al. further showed the successful integration capability of paper-based sensors into a fully autonomous and wearable ultralow-cost medical diagnostic system, referred to as the "Paper Watch," capable of continuously monitoring various vital signs such as heart rate, blood pressure, body temperature, and skin hydration. ${ }^{[147,152]}$

However, the use of such unconventional substrates can be still impractical for rugged applications involving extensive wear and tear, and requires optimized handling and techniques for efficient integration with the rest of the CMOS components. CMOS integration can take control of pumping, imaging, and processing thus being vital for the autonomous operation of an LOC system. Hence, when flexibility is needed, a better albeit expensive alternative to the unconventional paper substrates would be to the most commonly used CMOS platform, silicon, and overcome its brittleness through low-cost flexing mechanisms. As sensors are an integral part of any LOC system, Nassar et al. successfully showed the integration of multifunctional CMOS sensors on a large-scale flexible silicon platform. ${ }^{[153]}$ Their heterogeneous and monolithic integration approach uses only CMOS-compatible fabrication processes on bulk monocrystalline silicon (100). ${ }^{[153]}$ The sensors include tem- perature RTDs, pressure, strain, and humidity, which are all vital sensing elements needed for the characterization and understanding of fluid behavior inside microfluidic channels. This flexible silicon platform is compatible with the one-chip integration of silicon-based data processing units and interface circuitry required for a complete LOC system. Unlike the standard rigid silicon platforms that conventionally host CMOS sensors, the ability to combine sensors, actuators, microfluidic channels, and CMOS processing devices on a flexible silicon platform in a lowcost manner will reveal new opportunities in the field of portable and wearable biosensing LOC diagnostic systems. ${ }^{[28,154]}$

\section{Outlook and Concluding Remarks}

Microfluidics is an important emerging area and technology for enhanced healthcare. With the increasing global population, it is critical to make healthcare technology accessible to everyone. Developing countries lack access to modern laboratories, thus require POC diagnostic devices that are portable, user friendly, and do not require preprocessing of biological samples. Devices based upon microfluidics have already shown encouraging results in replacing various laboratory procedures like flow cytometry, PCR tests, immunoassays, and blood chemistry. An overview of such applications driven by progress in CMOS based microfluidics can be seen in Figure 12. We can see how this the versatile CMOS technology combined with the resourceful microfluidics techniques is shaping a healthier lifestyle for us, where different diagnostic tests can be performed in real time on the go. Moving ahead, we can see more potential applications arising as CMOS enabled technologies will help microfluidic devices to find applications in our everyday world. ${ }^{[155]}$ CMOS technology has played critical enabling role to empower us in the digital era. For many microfluidic technologies, CMOS technology can help with data management. Miniaturized CMOS electronics are the most reliable option for data processing, storage, and transmission, and thus their integration can enhance the functionality of microfluidic devices. The challenges to commercialization are component integration and fabrication standardization. ${ }^{[156]}$ We have extensively reviewed the component integration with CMOS based sensors and actuators, while there is a dire need for replacement of PDMS as the main material for fabrication as PDMS requires multiple processes that cannot be automated which have kept the cost of mass fabrication high. Going forward, we believe that sensors and actuators can be made with emerging materials based on 1D nanowires and tubes, and 2D atomic crystal structure materials. However, their cost-effectiveness and reliable manufacturability have to be ensured. The emergence of 3D printing technology has opened up an opportunity for rapid prototyping of enhanced capabilities, like bioprinting of artificial organs. To make the broad spectrum of healthcare technology accessible to everyone, it will be of paramount importance to have affordable low-cost microfluidic devices. In that regard, paper-based devices with a low-cost assembly could be critical enablers. We have discussed various microfluidic technologies focusing on DNA analysis, assembly, cell manipulation, diagnostics, and drug delivery. There are many more applications which are not discussed here. Critical 


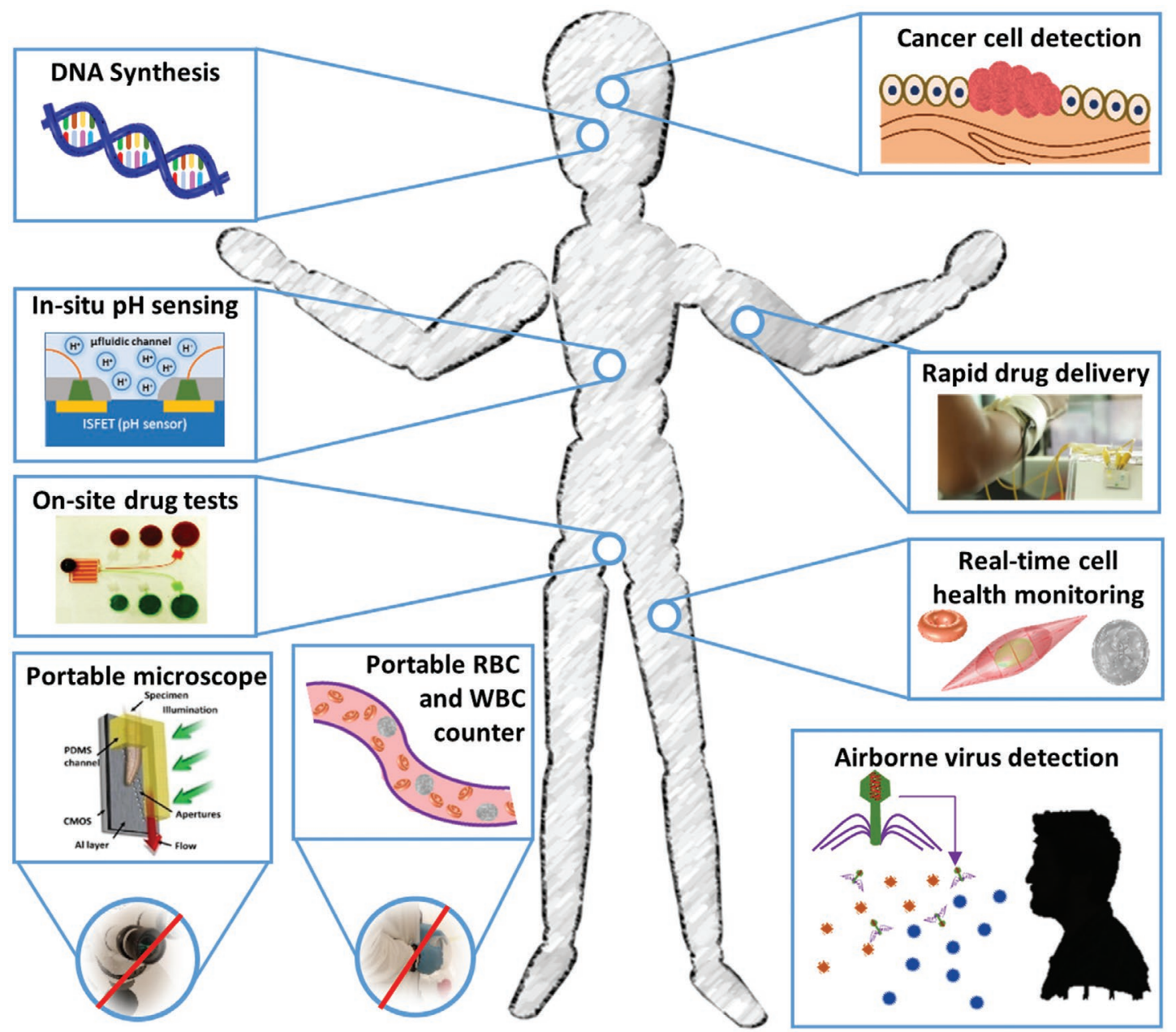

Figure 12. An illustration of how CMOS technology has facilitated microfluidic devices in replacing cumbersome laboratory processes and bulky lab equipment to portable PoC diagnostic devices. CMOS-based microfluidics has further enabled in-field DNA analysis, airborne virus detection, drug tests and delivery, real-time cell health monitoring, and cancer cells detection. Portable microscope image: reproduced with permission. ${ }^{\text {[78] }}$ Copyright 2010, National Academy of Sciences.

components, like sensors, actuators, and their fabrication and packaging, have been discussed and reviewed in detail. At the end, we also discussed some of our own contributions to ultralow-cost paper-based diagnostic devices and personalized medicinal platforms that allow users to prepare drugs and nutrients based on their genomic architecture and health conditions, as measured by various wearable healthcare gadgets. We see enormous potential for microfluidic technologies in affordable healthcare for everyone, and CMOS technology will play a major role in making that happen.

\section{Acknowledgements}

The authors deeply appreciate invaluable advice by William E. Ayer, Professor of Engineering Roger Howe of Stanford University. This publication is based upon work supported by the King Abdullah University of Science and Technology (KAUST) Office of Sponsored Research (OSR) under Award No. Sensor Innovation Initiative OSR-2015Sensors-2707, KAUST-KFUPM Special Initiative OSR-2016-KKI-2880, and Technology Transfer Office (TTO) under Award No. Proof of Concept GEN-01-4014. The authors also thank Veronica E. Tremblay, Editor,
Academic Writing, Office of the Vice President for Research, King Abdullah University of Science and Technology for editorial assistance.

\section{Conflict of Interest}

The authors declare no conflict of interest.

\section{Keywords}

CMOS, drug delivery, healthcare, image sensor, lab on chip, microfluidics, point-of-care

Received: October 3, 2017

Revised: November 19, 2017 Published online: February 27, 2018

[1] E. Ghafar-Zadeh, M. Sawan, IEEE Sens. J. 2008, 8, 325.

[2] G. M. Whitesides, Nature 2006, 442, 368. 
[3] a) A. Manz, D. J. Harrison, E. M. Verpoorte, J. C. Fettinger A. Paulus, H. Lüdi, H. M. Widmer, J. Chromatogr. A 1992, 593, 253; b) A. Khademhosseini, R. Langer, J. Borenstein, J. P. Vacanti, Proc. Natl. Acad. Sci. USA 2006, 103, 2480.

[4] P. S. Dittrich, K. Tachikawa, A. Manz, Anal. Chem. 2006, 78, 3887.

[5] N. Khalid, I. Kobayashi, M. Nakajima, Wiley Interdiscip. Rev.: Syst. Biol. Med. 2017, 9, e1381.

[6] a) A. Manz, N. Graber, H. á. Widmer, Sens. Actuators, B 1990, 1 , 244; b) D. Mark, S. Haeberle, G. Roth, F. von Stetten, R. Zengerle, Chem. Soc. Rev. 2010, 39, 1153; c) S. Haeberle, R. Zengerle, Lab Chip 2007, 7, 1094.

[7] P. Abgrall, A. Gue, J. Micromech. Microeng. 2007, 17, R15.

[8] a) C. J. Easley, J. M. Karlinsey, J. M. Bienvenue, L. A. Legendre, M. G. Roper, S. H. Feldman, M. A. Hughes, E. L. Hewlett, T. J. Merkel, J. P. Ferrance, Proc. Natl. Acad. Sci. USA 2006, 103, 19272; b) D. Erickson, S. Mandal, A. H. Yang, B. Cordovez, Microfluid. Nanofluid. 2008, 4, 33

[9] a) S. J. Reinholt, A. Behrent, C. Greene, A. Kalfe, A. J. Baeumner Anal. Chem. 2013, 86, 849; b) W. Jung, J. Han, J.-W. Choi, C. H. Ahn, Microelectron. Eng. 2015, 132, 46; c) P. Lisowski, P. K. Zarzycki, Chromatographia 2013, 76, 1201; d) Y. Zhu, Q. Fang, Anal. Chim. Acta 2013, 787, 24.

[10] Y. Huang, A. J. Mason, Lab Chip 2013, 13, 3929.

[11] H. Lee, Y. Liu, D. Ham, R. M. Westervelt, Lab Chip 2007, 7, 331.

[12] H. Norian, R. M. Field, I. Kymissis, K. L. Shepard, Lab Chip 2014 14, 4076.

[13] D.-H. Kuan, I.-S. Wang, J.-R. Lin, C.-H. Yang, C.-H. Huang, Y.-H. Lin, C.-T. Lin, N.-T. Huang, Lab Chip 2016, 16, 3105.

[14] E. Ghafar-Zadeh, M. Sawan, D. Therriault, S. Rajagopalan, V. P. Chodavarapu, Microelectron. Eng. 2009, 86, 2104

[15] D. Welch, J. B. Christen, J. Micromech. Microeng. 2013, 23, 035009

[16] T. Datta-Chaudhuri, E. Smela, P. A. Abshire, IEEE Trans. Biomed. Circuits Syst. 2016, 10, 1129.

[17] T. Datta-Chaudhuri, P. Abshire, E. Smela, Lab Chip 2014, 14, 1753.

[18] G. E. Moore, Proc. IEEE 1998, 86, 82.

[19] T. Voitsekhivska, E. Suthau, K.-J. Wolter, presented at 2014 IEEE 64th Electronic Components and Technology Conf. (ECTC), Orlando FL, USA May 2014

[20] J. Z. Chen, A. A. Darhuber, S. M. Troian, S. Wagner, Lab Chip 2004, $4,473$.

[21] G. Medoro, N. Manaresi, A. Leonardi, L. Altomare, M. Tartagni, R. Guerrieri, IEEE Sens. J. 2003, 3, 317

[22] A. Gao, X. Liu, T. Li, X. Gao, Y. Wang, J. Adhes. Sci. Technol. 2012, 26, 2165.

[23] D. Issadore, T. Franke, K. A. Brown, T. P. Hunt, R. M. Westervelt, J. Microelectromech. Syst. 2009, 18, 1220.

[24] a) D. Tseng, O. Mudanyali, C. Oztoprak, S. O. Isikman, I. Sencan, O. Yaglidere, A. Ozcan, Lab Chip 2010, 10, 1787; b) A. Ozcan, Lab Chip 2014, 14, 3187.

[25] T. Laksanasopin, T. W. Guo, S. Nayak, A. A. Sridhara, S. Xie, O. O. Olowookere, P. Cadinu, F. Meng, N. H. Chee, J. Kim, Sci. Transl. Med. 2015, 7, 273re].

[26] K. Yang, H. Peretz-Soroka, Y. Liu, F. Lin, Lab Chip 2016, 16, 943.

[27] a) J. P. Rojas, G. A. T. Sevilla, M. M. Hussain, Sci. Rep. 2013, 3, 2609; b) M. M. Hussain, A. M. Hussain, J. M. Nassar, A. T. Kutbee, A. Gumus, A. N. Hanna, presented at 2016 9th Int. Conf. Electrical and Computer Engineering (ICECE), Dhaka, Bangladesh December 2016; c) A. M. Hussain, M. M. Hussain, Adv. Mater. 2015, 28 , 4219; d) S. F. Shaikh, M. T. Ghoneim, G. A. T. Sevilla, J. M. Nassar A. M. Hussain, M. M. Hussain, IEEE Trans. Electron Devices 2017, 64, 1894.

[28] J. P. Rojas, G. A. Torres Sevilla, M. T. Ghoneim, S. B. Inayat, S. M. Ahmed, A. M. Hussain, M. M. Hussain, ACS Nano 2014, 8, 1468.

[29] A. Rasmussen, M. E. Zaghloul, presented at 42nd Midwest Symposium on Circuits and Systems, Las Cruces, NM, USA August 1999.
[30] M. Sawan, M. Miled, E. Ghafar-Zadeh, presented at Engineering in Medicine and Biology Society (EMBC), 2010 Annual International Conference of the IEEE, Buenos Aires, Argentina August-September 2010.

[31] Y. H. Ghallab, H. A. El-Hamid, Y. Ismail, IEEE Design Test 2015, 32, 20.

[32] Z. Liu, X. Han, L. Qin, Adv. Healthcare Mater. 2016, 5, 871.

[33] T. Bayraktar, S. B. Pidugu, Int. J. Heat Mass Transfer 2006, 49, 815.

[34] D. Malsch, M. Kielpinski, R. Merthan, J. Albert, G. Mayer, J. Köhler, H. Süße, M. Stahl, T. Henkel, Chem. Eng. J. 2008, 135, S166.

[35] A. Radenovic, Advanced Bioengineering Methods Laboratory, 2005 pp. 1-27.

[36] P. K. Challa, T. Kartanas, J. Charmet, T. P. Knowles, Biomicrofluidics 2017, 11, 014113

[37] N. Islam, S. Sayed, Microelectromechanical Systems and Devices, InTech, Rijeka, Croatia 2012.

[38] H. Becker, C. Gärtner, Anal. Bioanal. Chem. 2008, 390, 89.

[39] J. R. Anderson, D. T. Chiu, H. Wu, O. Schueller, G. M. Whitesides, Electrophoresis 2000, 21, 27.

[40] H. Becker, L. E. Locascio, Talanta 2002, 56, 267.

[41] U. M. Attia, S. Marson, J. R. Alcock, Microfluid. Nanofluid. 2009, 7, 1.

[42] H. Becker, U. Heim, Sens. Actuators, A 2000, 83, 130.

[43] J. Rossier, F. Reymond, P. E. Michel, Electrophoresis 2002, 23, 858.

[44] D. Mark, P. Weber, S. Lutz, M. Focke, R. Zengerle, F. von Stetten, Microfluid. Nanofluid. 2011, 10, 1279.

[45] J. Hruby, B. E. Carlsten, AIP Conf. Proc. 2002, 625, 55.

[46] J. Garra, T. Long, J. Currie, T. Schneider, R. White, M. Paranjape, J. Vac. Sci. Technol., A 2002, 20, 975.

[47] C. Iliescu, B. Chen, J. Miao, Sens. Actuators, A 2008, 143, 154.

[48] a) A. Aguirre-Pablo, J. Zhang, E. Li, S. Thoroddsen, presented at APS Meeting Abstracts, San Antonio, TX March 2015; b) J. M. Zhang, E. Q. Li, A. A. Aguirre-Pablo, S. T. Thoroddsen, RSC Adv. 2016, 6, 2793.

[49] a) X. Zhang, X. Han, T. E. Sarvey, C. E. Green, P. A. Kottke, A. G. Fedorov, Y. Joshi, M. S. Bakir, J. Electron. Packag. 2016, 138, 010910; b) J. M. Zhang, A. A. Aguirre-Pablo, E. Q. Li, U. Buttner, S. T. Thoroddsen, RSC Adv. 2016, 6, 81120 .

[50] N. Bhattacharjee, A. Urrios, S. Kang, A. Folch, Lab Chip 2016, 16, 1720.

[51] S. Waheed, J. M. Cabot, N. P. Macdonald, T. Lewis, R. M. Guijt, B. Paull, M. C. Breadmore, Lab Chip 2016, 16, 1993.

[52] R. Amin, S. Knowlton, A. Hart, B. Yenilmez, F. Ghaderinezhad, S. Katebifar, M. Messina, A. Khademhosseini, S. Tasoglu, Biofabrication 2016, 8, 022001

[53] A. Uddin, K. Milaninia, C.-H. Chen, L. Theogarajan, IEEE Trans. Compon., Packag., Manuf. Technol. 2011, 1, 1996.

[54] M. Crescentini, F. Thei, M. Bennati, S. Saha, M. R. de Planque, H. Morgan, M. Tartagni, IEEE Trans. Biomed. Circuits Syst. 2015, 9, 334.

[55] M. Khorasani, M. Behnam, L. van den Berg, C. J. Backhouse, D. G. Elliott, IEEE Trans. Biomed. Circuits Syst. 2009, 3, 89.

[56] C.-S. A. Gong, K. F. Lei, BioMed Res. Int. 2014, 2014, 734675.

[57] S.-S. Li, C.-M. Cheng, Lab Chip 2013, 13, 3782.

[58] E. Ghafar-Zadeh, M. Sawan, D. Therriault, Sens. Actuators, A 2008, $141,454$.

[59] C. Guiducci, C. Stagni, G. Zuccheri, A. Bogliolo, L. Benini, B. Samorı, B. Ricco, Biosens. Bioelectron. 2004, 19, 781.

[60] E. Ghafer-Zadeh, M. Sawan, presented at 2007 IEEE International Symposium on Circuits and Systems (ISCAS), New Orleans, LA, USA May 2007.

[61] E. Ghafar-Zadeh, M. Sawan, presented at IEEE International Workshop on Medical Measurements and Applications, 2008 (MeMeA 2008), Ottawa, ON, Canada May 2008.

[62] A. Romani, N. Manaresi, L. Marzocchi, G. Medoro, A. Leonardi, L. Altomare, M. Tartagni, R. Guerrieri, presented at 2004 IEEE International Solid-State Circuits Conference (Digest of Technical Papers, ISSCC), San Francisco, CA, USA February 2004. 
[63] Y. Ho, G. Wang, K. Y.-T. Lai, Y.-W. Lu, K.-M. Liu, Y.-M. Wang, C.-Y. Lee, presented at 2016 IEEE International Symposium on Circuits and Systems (ISCAS), Montreal, QC, Canada May 2016.

[64] D. Welch, J. B. Christen, presented at 2012 Annual International Conference of the IEEE Engineering in Medicine and Biology Society (EMBC), San Diego, CA, USA August-September 2012.

[65] S. B. Prakash, P. Abshire, presented at Sensors, 2005 IEEE, Irvine, CA, USA October-November 2005.

[66] A. Balasubramanian, B. Bhuva, R. Mernaugh, F. R. Haselton, IEEE Sens. J. 2005, 5, 340.

[67] J. Gomez-Quinones, S. Camacho-Leon, G. Dieck-Assad, S. O. Martinez-Chapa, presented at 2011 IEEE Electronics, Robotics and Automotive Mechanics Conference (CERMA), Cuernavaca, Morelos, Mexico November 2011.

[68] B. Van Dorst, M. Brivio, E. Van Der Sar, M. Blom, S. Reuvekamp, S. Tanzi, R. Groenhuis, A. Adojutelegan, E.-J. Lous, F. Frederix, Biosens. Bioelectron. 2016, 78, 126.

[69] J. H. Jung, C. Han, S. A. Lee, J. Kim, C. Yang, Lab Chip 2014, 14, 3781.

[70] R. R. Singh, L. Leng, A. Guenther, R. Genov, IEEEJ. Solid-State Circuits 2012, 47, 2822.

[71] N. Misawa, S. Takeuchi, IEEE Trans. Electr. Electron. Eng. 2011, 6, 97.

[72] E. R. Rodrigues, R. A. Lapa, Anal. Bioanal. Chem. 2010, 397, 381.

[73] R. R. Singh, L. Leng, A. Guenther, R. Genov, presented at SPIE NanoScience+ Engineering, San Diego, CA, USA August 2009.

[74] Y. Demircan, S. Örgüç, J. Musayev, E. Özgür, M. Erdem, U. Gündüz, S. Eminolu, H. Külah, T. Akın, presented at Transducers-2015 18th International Conference on Solid-State Sensors, Actuators and Microsystems (TRANSDUCERS), Toronto, ON, Canada August 2014.

[75] G. H. Hall, T. Ma, M. H. Couse, S. Hume, D. L. Sloan, D. G. Elliott, D. M. Glerum, C. J. Backhouse, presented at 2014 IEEE 14th International Conference on Nanotechnology (IEEE-NANO), Toronto, ON, Canada August 2014.

[76] H. Eltoukhy, K. Salama, A. E. Gamal, IEEE J. Solid-State Circuits 2006, 41, 651

[77] D. Lange, C. W. Storment, C. A. Conley, G. T. Kovacs, Sens. Actuators, B 2005, 107, 904.

[78] S. Pang, X. Cui, J. DeModena, Y. M. Wang, P. Sternberg, C. Yang, Lab Chip 2010, 10, 411.

[79] Y. Zhao, Z. S. Stratton, F. Guo, M. I. Lapsley, C. Y. Chan, S.-C. S. Lin, T. J. Huang, Lab Chip 2013, 13, 17.

[80] X. Cui, L. M. Lee, X. Heng, W. Zhong, P. W. Sternberg, D. Psaltis, C. Yang, Proc. Natl. Acad. Sci. USA 2008, 105, 10670.

[81] T. Wang, X. Huang, Q. Jia, M. Yan, H. Yu, K. S. Yeo, presented at 2012 IEEE Biomedical Circuits and Systems Conference (BioCAS), Hsinchu, Taiwan November 2012.

[82] M. Yan, X. Huang, Q. Jia, R. Nadipalli, T. Wang, Y. Shang, H. Yu, M. Je, K. Yeo, Proc. SPIE 2012, 8298, 829804.

[83] E. H. Doeven, G. J. Barbante, A. J. Harsant, P. S. Donnelly, T. U. Connell, C. F. Hogan, P. S. Francis, Sens. Actuators, B 2015, 216, 608.

[84] I. E. Araci, B. Su, S. R. Quake, Y. Mandel, Nat. Med. 2014, 20, 1074.

[85] J. Kong, Q. Wei, D. Tseng, J. Zhang, E. Pan, M. Lewinski, O. Garner, A. Ozcan, D. Di Carlo, ACS Nano 2017, 11, 2934.

[86] J. Hu, X. Cui, Y. Gong, X. Xu, B. Gao, T. Wen, T. J. Lu, F. Xu, Biotechnol. Adv. 2016, 34, 305.

[87] A. Vittoriosi, J. Brandner, P. Ruther, O. Paul, R. Dittmeyer, Exp. Heat Transfer 2014, 27, 389.

[88] P. A. Hammond, D. Ali, D. R. Cumming, IEEE Sens. J. 2004, 4, 706.

[89] E. Buitrago, M. Fernández-Bolaños, S. Rigante, C. F. Zilch, N. S. Schröter, A. M. Nightingale, A. M. Ionescu, Sens. Actuators, B 2014, 193, 400.

[90] a) P. Bergveld, Biosensors 1986, 2, 15; b) E. Stern, A. Vacic, M. A. Reed, IEEE Trans. Electron Dev. 2008, 55, 3119.
[91] M. Cao, S. K. Mahto, O. Yadid-Pecht, IEEE Sens. J. 2016, 16, 3611.

[92] D.-S. Kim, J.-E. Park, J.-K. Shin, P. K. Kim, G. Lim, S. Shoji, Sens. Actuators, B 2006, 117, 488

[93] K.-M. Chang, K.-Y. Chao, K.-F. Lin, Sens. Actuators, B 2008, 130, 943.

[94] N. Ward, X. Mu, G. Serrano, E. Covington, C. Kurdak, E. T. Zellers, A. J. Mason, W. Li, Micro Nano Lett. 2012, 7, 721.

[95] X. Mu, N. Ward, L. Li, W. Li, A. J. Mason, E. Covington, G. Serrano, C. Kurdak, E. Zellers, presented at 2012 IEEE International Symposium on Circuits and Systems (ISCAS), Seoul, South Korea May 2012.

[96] L. Y. Yeo, H. C. Chang, P. P. Chan, J. R. Friend, Small 2011, 7, 12.

[97] Y. Xu, X. Hu, J. Liang, J. Sun, W. Gu, T. Zhao, Z. Wen, Anal. Bioanal. Chem. 2009, 394, 1947.

[98] Y. Liu, H. Lee, R. M. Westervelt, D. Ham, presented at Proceedings of the Bipolar/BiCMOS Circuits and Technology Meeting, Santa Barbara, CA, USA October 2005.

[99] J. Martinez-Quijada, T. Ma, G. H. Hall, M. Reynolds, D. Sloan, S. Caverhill-Godkewitsch, D. M. Glerum, D. Sameoto, D. G. Elliott, C. J. Backhouse, J. Micromech. Microeng. 2015, 25, 075005.

[100] T. Voitsekhivska, E. Suthau, S. Kirsten, M. Schubert, F. Zörgiebel, G. Cuniberti, K.-J. Wolter, presented at 2014 IEEE 16th Electronics Packaging Technology Conference (EPTC), Singapore December 2014.

[101] R. Correia, S. Catarino, J. Rocha, G. Minas, presented at 39th Annual Conference of the IEEE Industrial Electronics Society (IECON 2013), Vienna, Austria November 2013.

[102] J. Clarke, H.-C. Wu, L. Jayasinghe, A. Patel, S. Reid, H. Bayley, Nat. Nanotechnol. 2009, 4, 265.

[103] C. Dekker, Nat. Nanotechnol. 2007, 2, 209.

[104] J. Kim, R. Maitra, K. D. Pedrotti, W. B. Dunbar, IEEE Trans. Biomed. Circuits Syst. 2013, 7, 285.

[105] M. Behnam, G. Kaigala, M. Khorasani, P. Marshall, C. Backhouse, D. Elliott, Lab Chip 2008, 8, 1524.

[106] H. Yin, L. Li, A. J. Mason, presented at 2016 IEEE International Symposium on Circuits and Systems (ISCAS), Montreal, QC, Canada May 2016.

[107] A. Wu, L. Wang, E. Jensen, R. Mathies, B. Boser, Lab Chip 2010, 10, 519.

[108] B. Zhang, Q. Dong, C. E. Korman, Z. Li, M. E. Zaghloul, Sci. Rep. 2013, 3, 1038.

[109] E. Ghafar-Zadeh, M. Sawan, M. Hajj-Hassan, M. Miled, presented at 50th Midwest Symposium on Circuits and Systems, 2007 (MWSCAS 2007), Montreal, Quebec, Canada August 2007.

[110] E. Ghafar-Zadeh, M. Sawan, D. Therriault, Sens. Actuators, A 2007, 134, 27.

[111] N. Halonen, J. Kilpijärvi, M. Sobocinski, T. Datta-Chaudhuri, A. Hassinen, S. B. Prakash, P. Möller, P. Abshire, S. Kellokumpu, A. L. Spetz, Beilstein J. Nanotechnol. 2016, 7, 1871.

[112] M. Birkholz, A. Mai, C. Wenger, C. Meliani, R. Scholz, Wiley Interdiscip. Rev.: Nanomed. Nanobiotechnol. 2015, 8, 355.

[113] C. B. Kaynak, M. Kaynak, M. Wietstruck, S. Marschmeyer, P. Kulse, K. Schulz, H. Silz, A. Kruger, R. Barth, K. Schmalz, presented at 2014 IEEE Topical Conference on Biomedical Wireless Technologies, Networks, and Sensing Systems (BioWireleSS), Newport Beach, CA, USA January 2014

[114] L. Li, A. Mason, presented at 2014 IEEE International Symposium on Circuits and Systems (ISCAS), Melbourne VIC, Australia June 2014

[115] M. Muluneh, D. Issadore, Lab Chip 2014, 14, 4552.

[116] H. Schäfer, L. Schöler, K. Seibel, M. Böhm, Appl. Surf. Sci. 2008, 255,646 .

[117] T. Datta-Chaudhuri, P. Abshire, E. Smela, presented at 2014 IEEE International Symposium on Circuits and Systems (ISCAS), Melbourne VIC, Australia June 2014.

[118] R. Das, P. Harrop, Cambridge: IDTechEx, IDTechEx 2013. 
[119] S. Xu, Y. Zhang, L. Jia, K. E. Mathewson, K.-I. Jang, J. Kim, H. Fu, X. Huang, P. Chava, R. Wang, Science 2014, 344, 70.

[120] P. Mostafalu, M. Akbari, K. A. Alberti, Q. Xu, A. Khademhosseini, S. R. Sonkusale, Microsyst. Nanoeng. 2016, 2, 16039.

[121] N. Manaresi, A. Romani, G. Medoro, L. Altomare, A. Leonardi, M. Tartagni, R. Guerrieri, IEEE J. Solid-State Circuits 2003, 38, 2297.

[122] S. Choi, M. Goryll, L. Y. M. Sin, P. K. Wong, J. Chae, Microfluid. Nanofluid. 2011, 10, 231.

[123] M. Barbaro, A. Bonfiglio, L. Raffo, A. Alessandrini, P. Facci, I. Barák, Sens. Actuators, B 2006, 118, 41.

[124] Y. Zheng, J. Nguyen, Y. Wei, Y. Sun, Lab Chip 2013, 13, 2464.

[125] L. Hartley, K. V. Kaler, O. Yadid-Pecht, IEEE Trans. Circuits Syst. 2007, 54, 99.

[126] E. P. Dupont, E. Labonne, Y. Maruyama, C. Vandevyver, U. Lehmann, M. A. Gijs, E. Charbon, Sens. Actuators, B 2012, 174, 609

[127] S. Spieth, O. Brett, K. Seidl, A. Aarts, M. Erismis, S. Herwik F. Trenkle, S. Tätzner, J. Auber, M. Daub, J. Micromech. Microeng. 2011, 21, 125001.

[128] L. Gervais, N. De Rooij, E. Delamarche, Adv. Mater. 2011, 23, H151.

[129] A. M. Ghaemmaghami, M. J. Hancock, H. Harrington, H. Kaji, A. Khademhosseini, Drug Discovery Today 2012, 17, 173.

[130] L. Garibyan, N. Avashia, J. Invest. Dermatol. 2013, 133, 1.

[131] K. Hatakeyama, T. Tanaka, M. Sawaguchi, A. Iwadate, Y. Mizutani, K. Sasaki, N. Tateishi, T. Matsunaga, Electrochemistry 2008, 76, 573.

[132] C. D. Ahrberg, A. Manz, B. G. Chung, Lab Chip 2016, 16, 3866.

[133] E. Schonbrun, S. S. Gorthi, D. Schaak, Lab Chip 2012, 12, 268.

[134] Y. Zheng, C. Jacquemod, M. Sawan, presented at 2013 IEEE International Symposium on Circuits and Systems (ISCAS), Beijing, China May 2013

[135] X. Liu, X. Huang, Y. Jiang, H. Xu, J. Guo, H. W. Hou, M. Yan, H. Yu, IEEE Trans. Biomed. Circuits Syst. 2017, 11, 794.

[136] K. Hsieh, B. S. Ferguson, M. Eisenstein, K. W. Plaxco, H. T. Soh, Acc. Chem. Res. 2015, 48, 911

[137] C. D. Chin, T. Laksanasopin, Y. K. Cheung, D. Steinmiller, V. Linder, H. Parsa, J. Wang, H. Moore, R. Rouse, G. Umviligihozo, Nat. Med. 2011, 17, 1015.

[138] S. Nayak, A. Sridhara, R. Melo, L. Richer, N. H. Chee, J. Kim, V. Linder, D. Steinmiller, S. K. Sia, M. Gomes-Solecki, Sci. Rep. 2016, 6, 35069 .

[139] M. Andrianova, O. Gubanova, N. Komarova, E. Kuznetsov, A. Kuznetsov, Electroanalysis 2016, 28, 1311

[140] J. El Fouladi, Z. Lu, Y. Savaria, S. Martel, presented at 29th Annual International Conference of the IEEE Engineering in Medicine and Biology Society, 2007 (EMBS 2007), Lyon, France August 2007.

[141] J. Liu, C. Yang, H. Wu, Z. Lin, Z. Zhang, R. Wang, B. Li, F. Kang, L. Shi, C. P. Wong, Energy Environ. Sci. 2014, 7, 3674.

[142] J. M. Nassar, M. D. Cordero, A. T. Kutbee, M. A. Karimi, G. A. T. Sevilla, A. M. Hussain, A. Shamim, M. M. Hussain, Adv. Mater. Technol. 2016, 1, 1600004.

[143] a) F. Güder, A. Ainla, J. Redston, B. Mosadegh, A. Glavan, T. Martin, G. M. Whitesides, Angew. Chem. 2016, 128, 5821; b) W.-J. Lan, X. U. Zou, M. M. Hamedi, J. Hu, C. Parolo, E. J. Maxwell, P. Bühlmann, G. M. Whitesides, Anal. Chem. 2014, 86, 9548; c) Q. Mei, H. Jing, Y. Li, W. Yisibashaer, J. Chen, B. N. Li, Y. Zhang, Biosens. Bioelectron. 2016, 75, 427.

[144] a) J. Lessing, A. C. Glavan, S. B. Walker, C. Keplinger, J. A. Lewis, G. M. Whitesides, Adv. Mater. 2014, 26, 4677; b) S. Kim, H. Ko C. Lee, M. Kim, K. S. Kim, Y. H. Lee, K. Shin, Y. H. Cho, Adv. Mater. 2016, 28, 9765; c) H. Shigemune, S. Maeda, Y. Hara, N. Hosoya, S. Hashimoto, IEEE/ASME Trans. Mechatron. 2016, 21, 2746.

[145] M. M. Hamedi, A. Ainla, F. Güder, D. C. Christodouleas, M. T. Fernández-Abedul, G. M. Whitesides, Adv. Mater. 2016, 28, 5054.

[146] a) C. Gaspar, T. Sikanen, S. Franssila, V. Jokinen, Biomicrofluidics 2016, 10, 064120; b) E. J. Maxwell, A. D. Mazzeo, G. M. Whitesides, MRS Bull. 2013, 38, 309.
[147] J. M. Nassar, K. Mishra, K. Lau, A. A. Aguirre-Pablo, M. M. Hussain, Adv. Mater. Technol. 2017, 2, 1600228.

[148] A. W. Martinez, S. T. Phillips, M. J. Butte, G. M. Whitesides, Angew. Chem., Int. Ed. 2007, 46, 1318

[149] A. W. Martinez, S. T. Phillips, G. M. Whitesides, E. Carrilho, Anal. Chem. 2010, 82, 3

[150] A. W. Martinez, S. T. Phillips, G. M. Whitesides, Proc. Natl. Acad. Sci. USA 2008, 105, 19606.

[151] a) Z. Nie, F. Deiss, X. Liu, O. Akbulut, G. M. Whitesides, Lab Chip 2010, 10, 3163; b) Z. Nie, C. A. Nijhuis, J. Gong, X. Chen, A. Kumachev, A. W. Martinez, M. Narovlyansky, G. M. Whitesides, Lab Chip 2010, 10, 477.

[152] J. M. Nassar, M. M. Hussain, IEEE Trans. Electron Devices 2017, 64, 2022.

[153] J. M. Nassar, G. A. T. Sevilla, S. J. Velling, M. D. Cordero, M. M. Hussain, presented at 2016 IEEE International on Electron Devices Meeting (IEDM), San Francisco, CA, USA December 2016.

[154] G. A. Torres Sevilla, M. T. Ghoneim, H. Fahad, J. P. Rojas, A. M. Hussain, M. M. Hussain, ACS Nano 2014, 8, 9850.

[155] S. Sharma, J. Zapatero-Rodríguez, P. Estrela, R. O'Kennedy, Biosensors 2015, 5, 577

[156] R. Lo, Adv. Health Care Technol. 2017, 3, 3.

[157] X. Huang, J. Guo, X. Wang, M. Yan, Y. Kang, H. Yu, PLoS One 2014, 9, e104539.

[158] S. A. Lee, C. Yang, Lab Chip 2014, 14, 3056

[159] Z. Göröcs, A. Ozcan, IEEE Rev. Biomed. Eng. 2013, 6, 29.

[160] V. Marx, Nat. Methods 2015, 12, 393.

[161] J. Batovska, S. E. Lynch, B. C. Rodoni, T. I. Sawbridge, N. O. Cogan, J. Virol. Methods 2017, 249, 79.

[162] M. Kühnemund, Q. Wei, E. Darai, Y. Wang, I. Hernández-Neuta, Z. Yang, D. Tseng, A. Ahlford, L. Mathot, T. Sjöblom, Nat. Commun. 2017, 8, 13913.

[163] J. A. Russell, B. Campos, J. Stone, E. M. Blosser, N. BurkettCadena, J. L. Jacobs, bioRxiv 2017, 183780.

[164] http://www.alere-i.com/en/index.html (accessed: November 2017).

[165] https://usdiagnostics.roche.com/en/point-of-care-testing/ poc-testing/infectious-disease/cobas-liat-pcr-system.html (accessed: November 2017)

[166] http://www.coaguchek-usa.com/coaguchek_hcp/en_US/home/ coaguchek-products/coaguchek-mpoc.html (accessed: November 2017)

[167] https://usdiagnostics.roche.com/en/point-of-care-testing/ poc-testing/blood-glucose/accu-chek-inform-ii.html\# explore (accessed: November 2017).

[168] https://usdiagnostics.roche.com/en/point-of-care-testing/poctesting/urinalysis/urysis-1100.html (accessed: November 2017).

[169] https://usdiagnostics.roche.com/en/point-of-care-testing/ poc-testing/cholesterol/accutrend-plus.html\#overview (accessed: $11 / 15 / 2017)$

[170] http://www.bio-rad.com/en-ca/product/tc20-automated-cellcounter (accessed: November 2017).

[171] http://www.nanocellect.com/ (accessed: November 2017).

[172] http://chipcare.ca/the-pax-platform/ (accessed: November 2017).

[173] A. I. Barbosa, P. Gehlot, K. Sidapra, A. D. Edwards, N. M. Reis, Biosens. Bioelectron. 2015, 70, 5.

[174] O. Tokel, U. H. Yildiz, F. Inci, N. G. Durmus, O. O. Ekiz, B. Turker, C. Cetin, S. Rao, K. Sridhar, N. Natarajan, Sci. Rep. 2015, 5, 9152.

[175] https://www.elveflow.com/microfluidic-flow-control-products/ microfluidic-flow-control-module/microfluidic-liquid-flow-throughpressure-sensor (accessed: November 2017).

[176] http://www.microliquid.com/\#Automated-Instrumentation (accessed: November 2017). 